\title{
Overexpression of PtrABF gene, a bZIP transcription factor isolated from Poncirus trifoliata, enhances dehydration and drought tolerance in tobacco via scavenging ROS and modulating expression of stress-responsive genes
}

Xiao-San Huang ${ }^{1}$, Ji-Hong Liu ${ }^{1 *}$, Xue-Jun Chen ${ }^{2}$

\begin{abstract}
Background: Drought is one of the major abiotic stresses affecting plant growth, development and crop productivity. ABA responsive element binding factor (ABF) plays an important role in stress responses via regulating the expression of stress-responsive genes.

Results: In this study, a gene coding for ABF (PtrABF) was isolated from Poncirus trifoliata (L.) Raf. PtrABF had a complete open reading frame of 1347 bp, encoding a 448 amino acid peptide, and shared high sequence identities with ABFs from other plants. PtrABF was subcellularly targeted to the nucleus, exhibited transactivation activity in yeast cell and could bind to $A B R E$, supporting its role as a transcription factor. Expression levels of PtrABF were induced by treatments with dehydration, low temperature and ABA. Ectopic expression of PtrABF under the control of a CaMV 35 S promoter in transgenic tobacco plants enhanced tolerance to both dehydration and drought. Under dehydration and drought conditions, the transgenic plants accumulated lower levels of reactive oxygen species compared with wild type, accompanied by higher activities and expression levels of three antioxidant enzymes. In addition, steady-state mRNA levels of nine stress-responsive genes coding for either functional or regulatory proteins were induced to higher levels in the transgenic lines with or without drought stress.
\end{abstract}

Conclusions: PtrABF is a bZIP transcription factor and functions in positive modulation of drought stress tolerance. It may be an important candidate gene for molecular breeding of drought- tolerant plants.

\section{Background}

Plants are frequently threatened by drought that affects growth and development, productivity and quality [1]. Therefore, improvement of drought tolerance has been the subject of intense studies via either traditional breeding or biotechnology-mediated approach. As a first step towards improvement of drought tolerance via genetic engineering in plant, genes with functions in drought tolerance should be cloned and characterized. It

\footnotetext{
* Correspondence: liujihong@mail.hzau.edu.cn

${ }^{1}$ National Key Laboratory of Crop Genetic Improvement, Key Laboratory of Horticultural Plant Biology of Ministry of Education, Huazhong Agricultural University, Wuhan 430070 China

Full list of author information is available at the end of the article
}

has been established that plants respond and adapt to drought stress through modifying transcriptional levels of a large number of genes [2,3]. These genes can be generally classified into two main groups based on their products, effector molecules or regulator molecules, which are also correspondingly designated as delayedresponse genes or early-response genes based on their response timing [4-6]. Products of the former group function directly in protecting cells against damage derived from stresses and sustaining cell viability, and the latter is composed of regulatory proteins, such as transcription factors (TFs), protein kinases and protein phosphatases, that are responsible for transducing stress 
signaling and regulating expression of stress-responsive genes [5].

TF constitutes the key regulon that plays crucial roles in various biological processes, including stress response, through binding to the cis-acting element in the promoters of target genes [7]. ABA-responsive element (ABRE, PyACGTGGC), an important cis-element, is implicated in the transcriptional regulation of ABA- and/or stressresponsive genes [8]. In plants, $A B R E$ binding factors (ABFs)/ABRE binding proteins (AREBs) are basic region/leucine zipper (bZIP) class TFs $[9,10]$ that interact with ABRE. ABF/AREB family TFs have been cloned and characterized in Arabidopsis thaliana and other plants [11-15]. In the Arabidopsis genome, nine ABF/ AREB homologues have been identified so far $[16,17]$. Up-regulation of ABF/AREB family members by various abiotic stresses, such as drought, high salinity, cold, anoxia, and by ABA in different plants suggests that they are involved in $\mathrm{ABA}$ and/or stress signaling $[11,12,14,16,17]$.

TFs are known as master switch in plant stress response, and genetic transformation of the genes encoding TFs has been suggested as a possible approach for engineering stress tolerance as manipulation of a single TF can alter expression of a wide array of target genes [7]. Genetic transformation of ABF/AREB has been shown to render stress tolerance. For example, constitutive overexpression of $A R E B 1 / A B F 2, A B F 3$ or $A B F 4$ in Arabidopsis resulted in enhanced drought tolerance $[14,18]$. In another work, interestingly, overexpression of ABF2 in Arabidopsis was found to promote tolerance to drought, high salt, heat and oxidative stresses [19]. Later, ABF/AREB family members have been transformed into lettuce and Agrostis mongolica, and the transgenic plants were more tolerant to drought and cold/heat stresses than wild type [20,21]. All of these successful examples indicate that genetic manipulation of ABF/AREB family members can be of significance for modifying plant stress response [22].

Although ABF/AREB members have been comprehensively studied in the model plants, information concerning their counterparts in woody plants is relatively lacking. Trifoliate orange (Poncirus trifoliata (L.) Raf), an important rootstock for citrus, is drought intolerant, which has restricted its use in regions with water deficit. A raised question is whether or not the $\mathrm{ABF}$ homologue in trifoliate orange can function in stress tolerance. On the other hand, it was noticed that limited information is available concerning physiological and/or molecular basis on enhanced stress tolerance in the transgenic plants overexpressing an ABF gene in previous reports. Therefore, we tired to clone an $\mathrm{ABF}$ gene from trifoliate orange, and functionally characterize its role in dehydration/drought tolerance. In addition, accumulation of reactive oxygen species (ROS) and expression of stressresponsive genes in the transgenic plants were illustrated.

\section{Results}

\section{Cloning and bioinformatics analysis of PtrABF}

Eighteen sequences were found in the search of the citrus EST database, which were successfully combined into a contig. RT-PCR using the gene specific primers (GSP1) designed based on the contig gave rise to a single band. Sequencing and bioinformatics analysis showed that the cDNA, $1511 \mathrm{bp}$ in length, contained a 1347-bp open reading frame (ORF), along with a 35-bp 5' untranslated region (UTR) and a 129-bp 3' UTR. The cDNA, designated as PtrABF (Poncirus trifoliata $A B F$ ), encodes a predicted polypeptide of 448 amino acids with a calculated molecular weight of $48 \mathrm{kDa}$ and a pI of 9.66. This gene has been deposited in NCBI under the accession number of HM171703. Multiple alignments between PtrABF and eight other ABF proteins revealed high sequence identity among each other (Figure 1). At the C-terminus of PtrABF there was a bZIP signature (from position 366 to 424, E-value 3.98e-09) composed of a 25 -amino acid basic region and a leucine zipper containing three leucine repeats (at positions 396, 403 and 410) that were spaced from each other by seven amino acids. The proteins had four highly conserved regions, three at the $\mathrm{N}$-terminus (designated as $\mathrm{C} 1, \mathrm{C} 2$ and $\mathrm{C} 3$ ) and one at the end of C-terminus (C4). The phylogenetic tree constructed based on amino acid sequences of PtrABF and ABFs from other 13 representative plants showed that PtrABF was most closely related to its counterpart of grape, while it is most distant to rice (Figure 2).

\section{Expression pattern of PtrABF under various treatments}

In plants, an important feature of $A B F s$ is the induction of expression by various abiotic stresses. In order to know if PtrABF acts in the similar way, expression profiles of PtrABF under different treatment (stresses or ABA) were assessed via QRT-PCR. The transcript level of PtrABF began to accumulate $1 \mathrm{~h}$ after dehydration, and increased progressively until the end of treatment, which was more than 13 folds of the initial level (Figure 3A). Steady-state mRNA of PtrABF began to accumulate $5 \mathrm{~h}$ after low temperature treatment, and continued to increase until it reached the highest level at $3 \mathrm{~d}$ (Figure 3B). When subjected to salt treatment, the transcript of PtrABF did not change notably except a slight decrease at $5 \mathrm{~h}$ and $1 \mathrm{~d}$ (Figure 3C), indicating that PtrABF was not salt-inducible. In the case of ABA treatment, mRNA abundance was first reduced at $1 \mathrm{~d}$, resumed to the basal level during the next two days, and increased at 4 $\mathrm{d}$ and maintained thereafter (Figure 3D). 


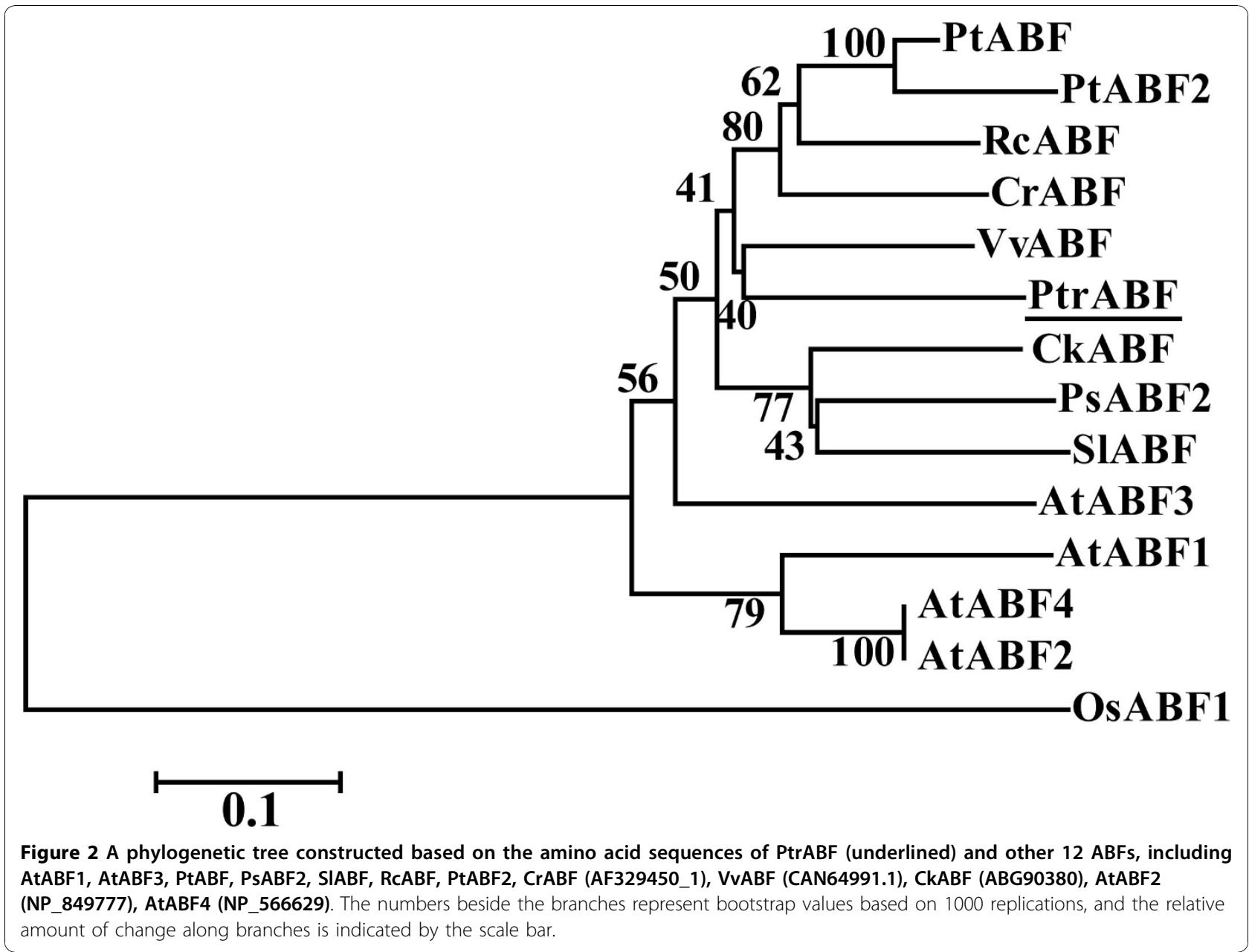

PtrABF was localized in the nucleus

Sequence analysis showed that there was a nuclear localization signal (from position 359 to 376) in the basic region of the bZIP DNA binding domain, implying that PtrABF may be localized in the nucleus. In order to verify this, subcellular localization of PtrABF was examined by monitoring the GFP fluorescence in the onion epidermis cells transformed with either the fusion construct (PtrABF-GFP) or the control (GFP). When onion cells were transformed with GFP plasmid, green fluorescence signals were observed in the entire cell region (Figure 4A-C). In contrast, fluorescence was exclusively detected in the nucleus of cells transformed with the fusion plasmid (Figure 4D-F), implying that PtrABF is a nuclear protein.

\section{PtrABF had transactivation activity and could bind to ABRE}

Transactivation activity is another defining feature for a transcription factor in addition to nuclear localization. To identify if PtrABF functions as a transcriptional activator the yeast two-hybrid analysis was used. For this purpose, the full-length PtrABF coding region was fused to the DNA-binding domain of GAL4 to generate a fusion plasmid, which was transformed into yeast and growth of the cells was compared with those transformed with the control plasmid (pGBKT7) on the same medium. Yeast cells carrying either the control plasmid or the fusion plasmid grew well on SD/-Leu/-Trp medium, indicating that the analysis system was reliable. On the $\mathrm{SD} /$-Ade/-His/-Leu/-Trp medium, the cells transformed with the control plasmid could not grow, whereas those transformed with the fusion plasmid grew normally. The yeast cells transformed with the fusion plasmid could also live on the medium added with 3AT, but the growth was repressed in a concentrationdependent manner (Figure 5A). In addition, when the yeast cells were cultured on the SD/-Ade/-His/-Leu/Trp medium added with both $15 \mathrm{mM} \mathrm{3-AT}$ and $20 \mathrm{mM}$ $\mathrm{X}-\alpha-\mathrm{Gal}$, only those transformed with the fusion plasmid turned blue (Figure 5B). All of these results indicated that PtrABF had transactivation activity in yeast. 

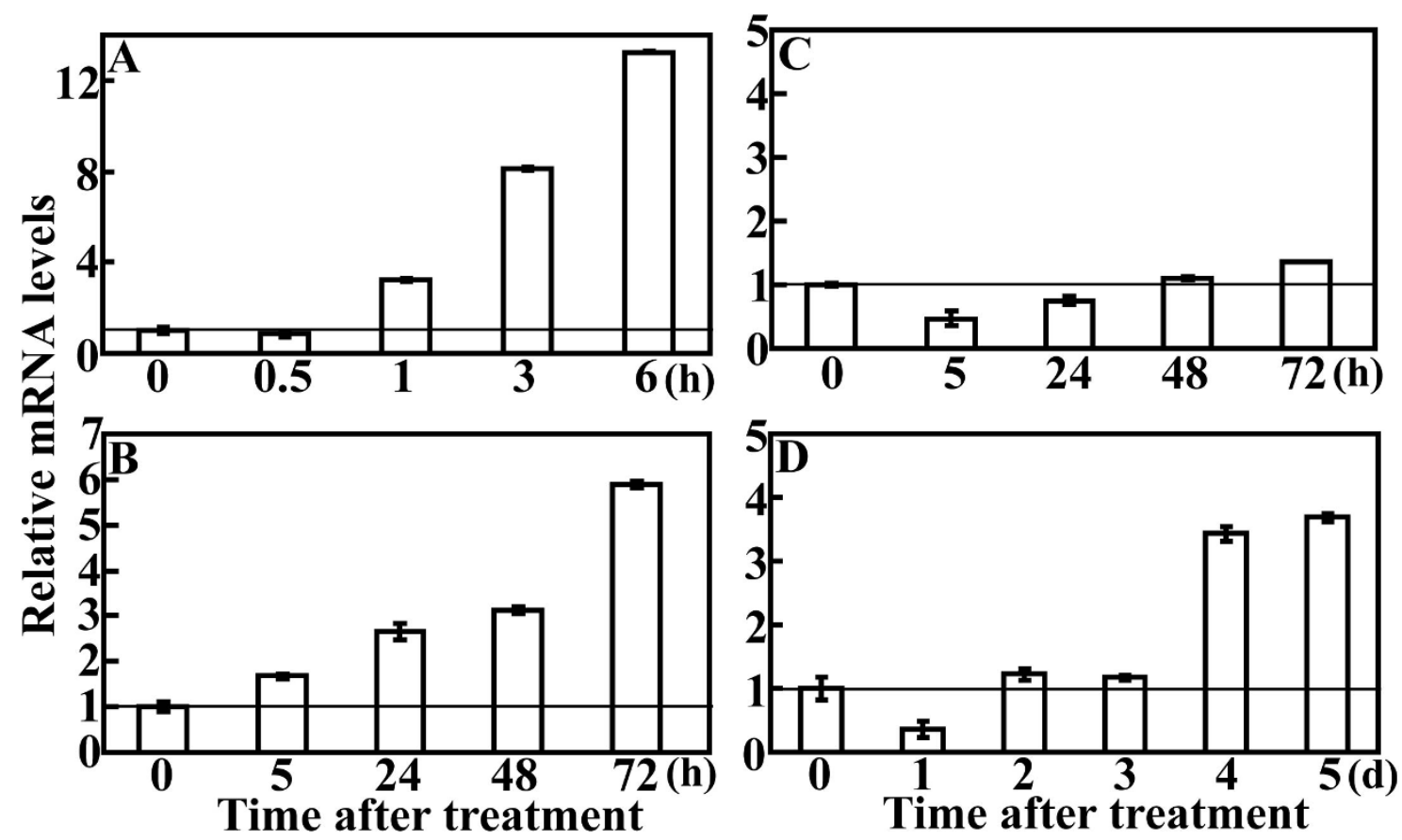

Figure 3 Time-course expression levels of PtrABF in trifoliate orange shoots under different treatments with dehydration (A), low temperature (B), salt (C) and ABA (D). Total RNA extracted from leaves of the treated shoots sampled at the indicated time was reversetranscribed to synthesize CDNAs, which were used for quantitative real time PCR analysis. For each treatment, the expression level at time point 0 was defined as 1.0, and data represented means \pm SE of three replicates.
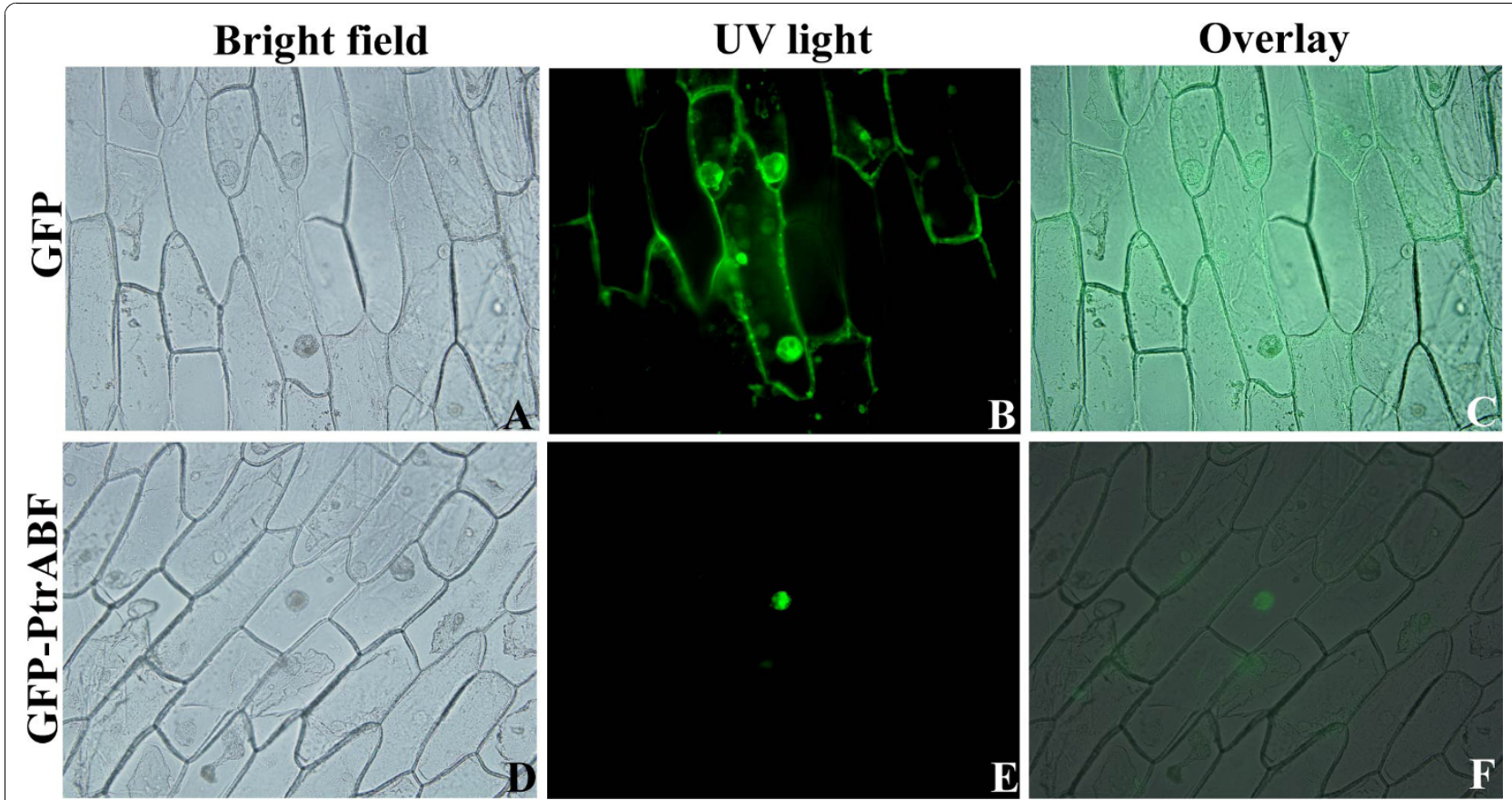

Figure 4 Subcellular localization of PtrABF via Agrobacterium-mediated transformation of onion epidermis. Fusion construct (PtrABF GFP) and control plasmid (GFP) were separately transformed into onion epidermal cells via Agrobacterium infection. Bright-field images (A, D), fluorescence images $(B, E)$, and the merged images $(C, F)$ of representative cells expressing GFP $(A, B$ and $C)$ or PtrABF: GFP fusion protein (D, E and F) are shown. 


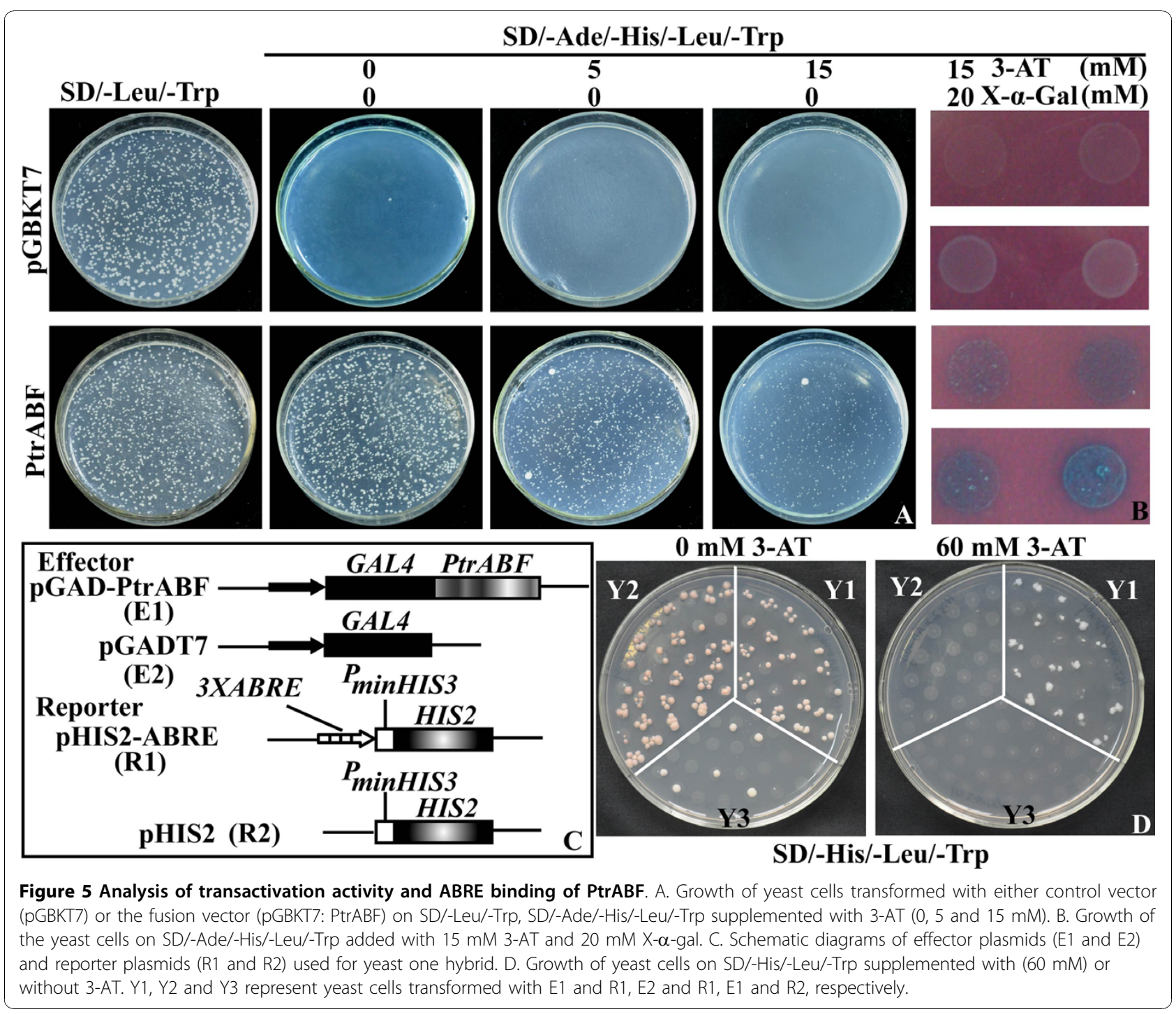

Previous work showed that ABF can bind to the ciselement ABRE [8]. In order to investigate whether PtrABF has the same DNA-binding activity, the full length ORF of PtrABF was fused to the GAL4 activation domain of vector pGADT7 and the fused construct (pGADT7-PtrABF) was co-transformed with pHISABRE construct containing triple tandem repeats of ABRE into yeast strain Y187 (Figure 5C). Although all of the yeast cells with different constructs could grow on SD/Leu-/Trp-/His medium without 3-AT, only the cells co-transformed with pGADT7-PtrABF and pHISABRE grew normally in the presence of 3-AT, while the growth of transformants containing constructs lacking either PtrABF or ABRE was completely inhibited (Figure $5 \mathrm{D})$, suggesting that PtrABF could bind to the ABRE and activate the reporter gene in yeast.
Overexpression of PtrABF enhances tolerance to dehydration and drought

To investigate the function of PtrABF, Agrobacteriummediated transformation of tobacco leaf discs was carried out using a binary vector containing PtrABF under the control of $35 \mathrm{~S}$ promoter of cauliflower mosaic virus (CaMV 35S). Totally, 9 T0 lines were characterized by PCR with primers specific to CaMV $35 \mathrm{~S}$ and PtrABF and NPTII, and 7 out of them were confirmed as putative transgenic lines, and overexpression of $\operatorname{Ptr} A B F$ in two lines (\#4 and \#19) was verified by semi-quantitative RT-PCR analysis (data not shown). These two lines and wild type (WT) were subjected to dehydration and drought treatment to investigate the role of PtrABF in water stress response. No morphological difference was noted between the transgenic lines and WT (data not 

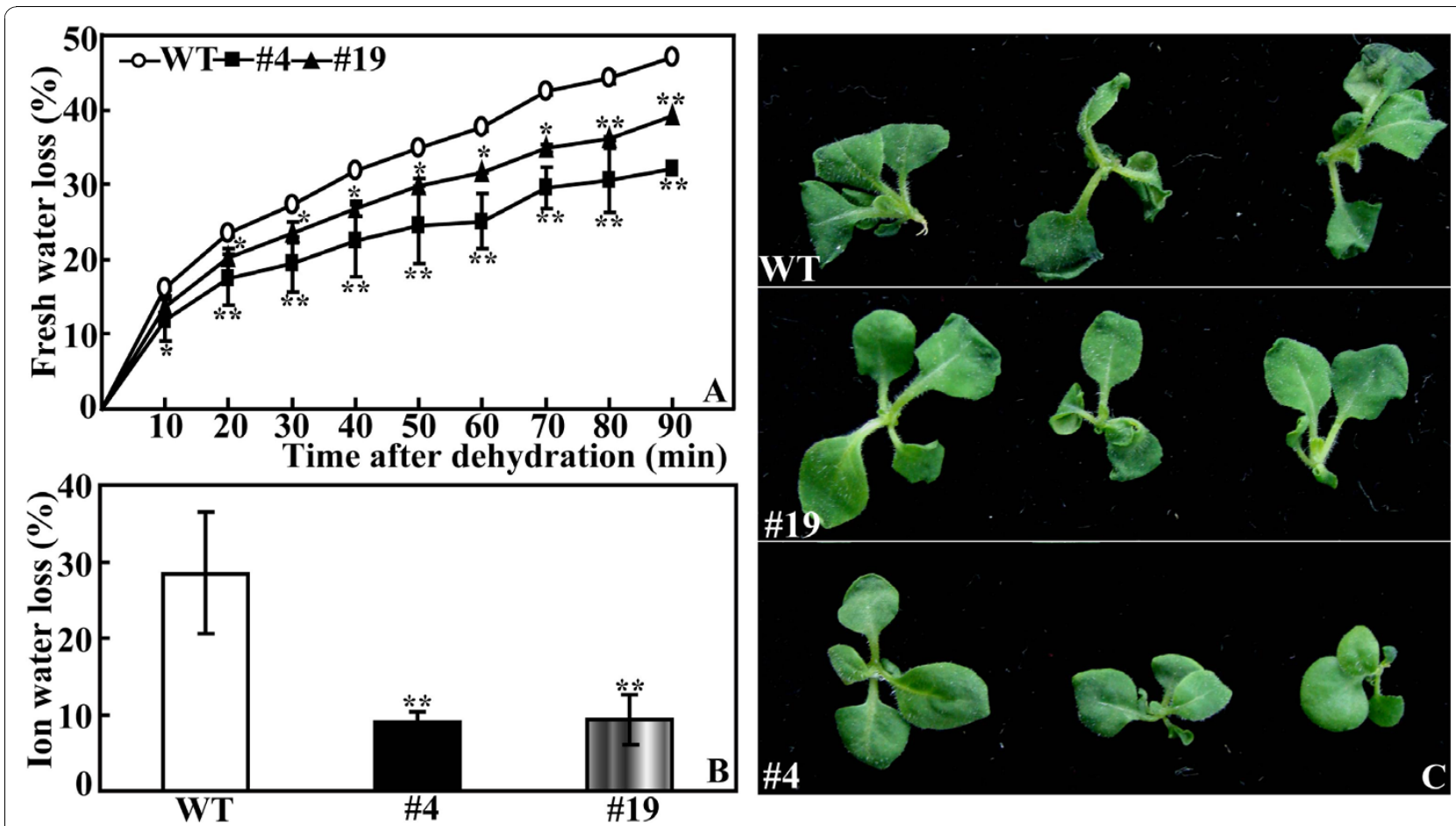

Figure 6 Phenotype and dehydration tolerance of wild type (WT) and transgenic lines (\#4 and \#19). (A) Time-course fresh water loss of WT, \#4 and \#19 during a 90-min dehydration. Aerial parts of 35-d-old in vitro seedlings were dehydrated at ambient environment, and the fresh weight (FW) was measured at the indicated time. Water loss was calculated by the decrease of FW relative to that at time 0. (B) Electrolyte leakage of WT, \#4 and \#19 after dehydration for 90 min. ${ }^{*}$ and ${ }^{* *}$ indicate that values of the two transgenic lines were significantly different from those of WT at $P<0.05$ and $p<0.01$, respectively. (C) Representative photographs of dehydrated WT (upper panel), \#4 (middle panel) and \#19 (lower panel)

shown). When the aerial parts of 5 -week-old in vitro seedlings were dehydrated, fresh water loss was increased in the two transgenic lines and WT with the progressing of dehydration. However, significantly higher water loss was observed in WT than the two transgenic lines from 10 min onwards (Figure 6A). Although \#19 lost less water than \#4, the difference was not statistically significant except at 60 and 90 min after dehydration. Electrolyte leakage of \#4 was equal to that of \#19, both of which were significantly smaller than WT (Figure 6B). When dehydration was completed, leaves of WT exhibited more serious wilting relative to the transgenic lines (Figure 6C).

Apart from dehydration, long-term water stress (drought) tolerance of the potted plants was also examined by water withholding. Morphological difference became apparent after watering was stopped for $7 \mathrm{~d}$, when WT lost its turgor while \#4 and \#19 grew better (Figure 7A). When the drought was extended to as long as 3 weeks, phonotypical difference was more dramatic between WT and the transgenic plants, as manifested by presence of more dead leaves in the former (Figure 7B). Electrolyte leakage of \#4 (16.7\%) and \#19 (22.3\%) was significantly lower in comparison with $42.3 \%$ of wile type (Figure 7C), whereas total chlorophyll of the trans-

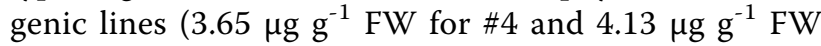
for \#19) was significantly higher than WT $\left(2.42 \mu \mathrm{g} \mathrm{g}^{-1}\right.$ FW, Figure 7D). The above results showed that the two transgenic lines were more tolerant to short-term dehydration shock or long-term water stress (drought).

\section{Accumulation of $\mathrm{O}_{2}-$ and $\mathrm{H}_{2} \mathrm{O}_{2}$ in the transgenic lines and} WT under drought

As the transgenic lines were more tolerant to dehydration and drought than wild type, attempts were made to compare accumulation of $\mathrm{H}_{2} \mathrm{O}_{2}$ and $\mathrm{O}_{2-}$, two main reactive oxygen species (ROS), in their leaves sampled at the end of water stress. For this purpose, leaves of the transgenic lines and wild type that have been either dehydrated for $90 \mathrm{~min}$ or exposed to drought for $21 \mathrm{~d}$ were stained with $\mathrm{DAB}$ or NBT to reveal in situ accumulation of $\mathrm{H}_{2} \mathrm{O}_{2}$ and $\mathrm{O}_{2^{-}}$, respectively. Histochemically staining showed that before dehydration, $\mathrm{H}_{2} \mathrm{O}_{2}$ and $\mathrm{O}_{2}$ - could also be stained, and the transgenic lines had slightly lower ROS levels relative to WT. Dehydration resulted in notable increased of ROS levels in both wild type and the two transgenic lines, whereas WT accumulated remarkably more $\mathrm{O}_{2}$ - and $\mathrm{H}_{2} \mathrm{O}_{2}$ than both \#4 and \#19, 


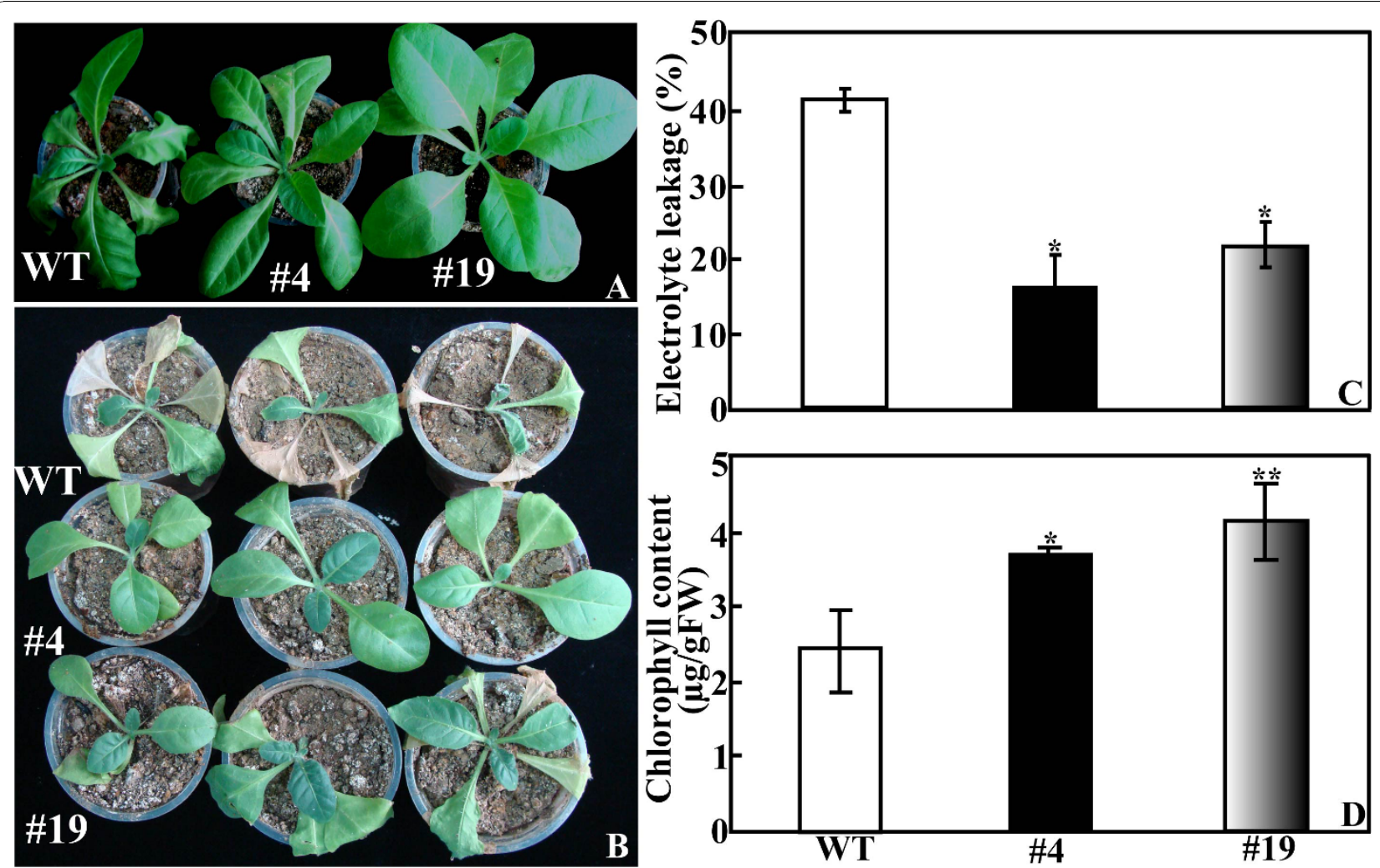

Figure 7 Phenotype and drought tolerance of wild type (WT) and transgenic lines (\#4 and \#19). (A-B) Representative photographs of potted plants of WT, \#4 and \#19 that have been exposed to water stress for $7 \mathrm{~d}(\mathrm{~A})$ and $21 \mathrm{~d}(\mathrm{~B})$. (C-D) Electrolyte leakage (C) and total chlorophyll content (D) of WT, \#4 and \#19 after $7 \mathrm{~d}$ of water stress. ${ }^{*}$ and ${ }^{*}$ indicate that values of the two transgenic lines were significantly different from those of WT at $P<0.05$ and $P<0.01$, respectively.

as less blue (Figure $8 \mathrm{~A}$ ) or brown (Figure $8 \mathrm{~B}$ ) products were observed in the latter two. Similarly, under drought the two transgenic lines accumulated dramatically less $\mathrm{O}_{2}$ - and $\mathrm{H}_{2} \mathrm{O}_{2}$ than WT (Figure $8 \mathrm{C}$ ), implying that less ROS was produced in the transgenic lines under the stressful conditions.

\section{Analysis of activity of antioxidant enzymes and expression levels of the encoding genes in transgenic lines and WT before and after drought}

The above-mentioned results demonstrated that the two transgenic lines produced less ROS compared with WT when they were simultaneously stressed. As ROSscavenging enzymes play critical role in detoxifying ROS activities of three key enzymes, superoxide (SOD), catalase (CAT) and peroxidase (POD), were measured in the two transgenic lines and WT before and $7 \mathrm{~d}$ after drought. Before water stress, activities of the three enzymes were higher than those of the control, but the difference was not prominent. Water stress caused decrease of SOD activity, which was significantly lower in WT than in \#4 and \#19 (Figure 9A). Exposure to drought resulted in slight rise of POD activity in WT, which was notably enhanced in the two transgenic lines. As a result, POD activity of \#4 and \#19 was 1.5 and 3 folds of that in WT, respectively (Figure 9B). Activity of CAT was augmented in all of the tested samples, while the transgenic lines had significantly higher activities than WT (Figure 9C). All of these showed that activities of the three detoxifying enzyme were higher in the transgenic lines than WT, in reverse proportion to the ROS accumulation of these lines. Examination of the genes encoding SOD, CAT and APX showed that before water stress the expression levels of the three genes were higher in the transgenic lines than in WT. Exposure to drought led to induction of these genes in both WT and the transgenic plants, whereas the transcript levels were still higher in the latter except NtAPX in \#19.

\section{Expression analysis of stress-responsive genes before and after drought}

Semi-quantitative RT-PCR was used to assess expression levels of nine stress-responsive genes in both WT and the transgenic lines under normal conditions and after 7 - $d$ water stress (Figure 10). In the absence of water 

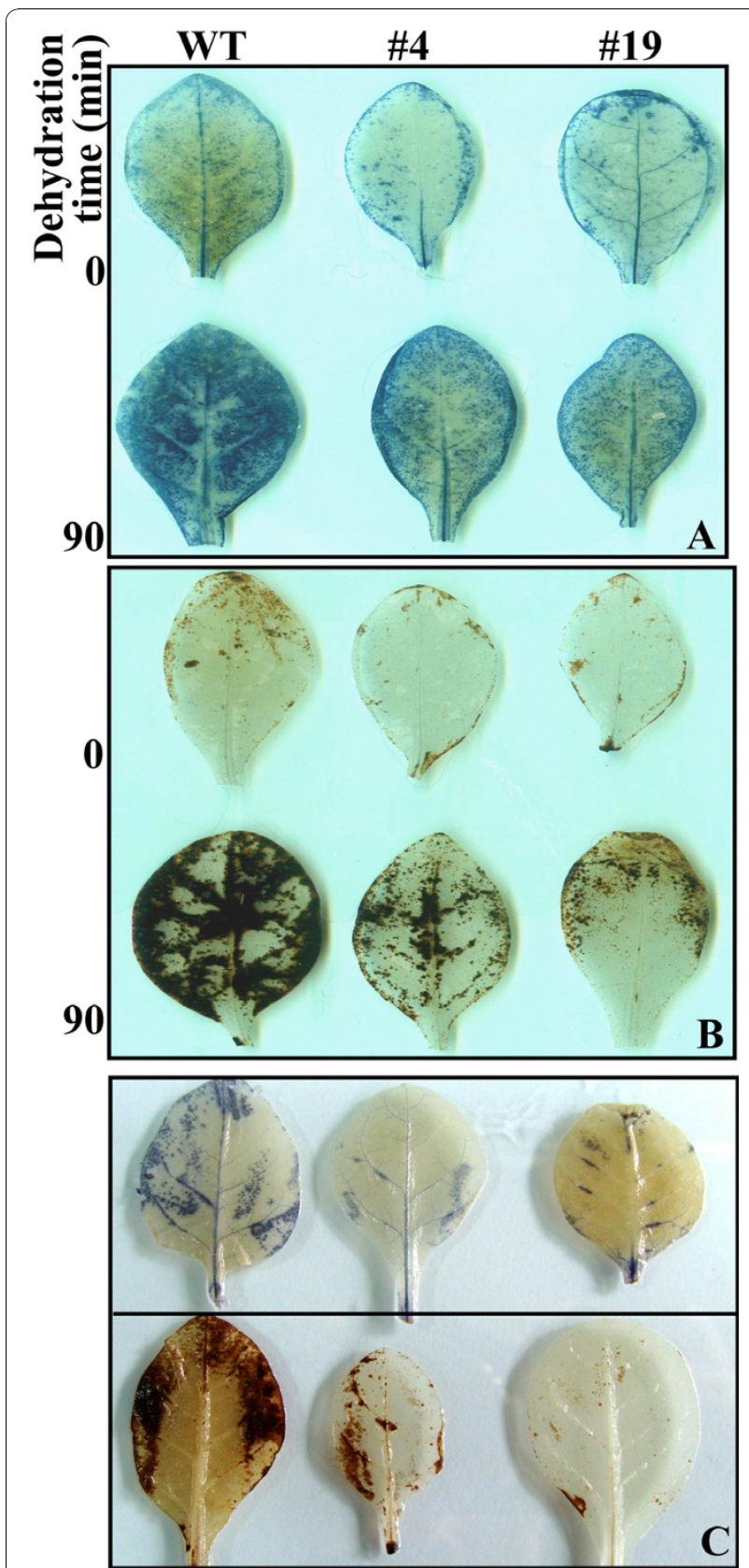

Figure 8 Histochemical staining by nitro blue tetrazolium (NBT) and diaminobenzidine (DAB) to reveal accumulation of $\mathrm{O}_{2}-$ and $\mathrm{H}_{2} \mathrm{O}_{2}$ in leaves of wild type (WT), transgenic lines (\#4 and \#19) subjected to $90-\mathrm{min}$ dehydration or 21 -d water stress. (A-B) Representative photos showing accumulation of $\mathrm{O}_{2^{-}}(\mathrm{A})$ and $\mathrm{H}_{2} \mathrm{O}_{2}$ (B) in leaves before (0) and after 90-min (90) dehydration. (C) Representative photos showing accumulation of $\mathrm{O}_{2}$ - (upper panel) and $\mathrm{H}_{2} \mathrm{O}_{2}$ (lower panel) in leaves of plants after 21- d water stress.

stress, transcript levels of NtADC1, NtADC2, NtSAMDC, $N t L E A 5, N t E R D 10 C, N t C D P K 2$ and NtDREB were obviously enhanced in the two transgenic lines compared with those of WT, whereas those of NtAREB and $N E E R F$ underwent minor change. Drought stress caused

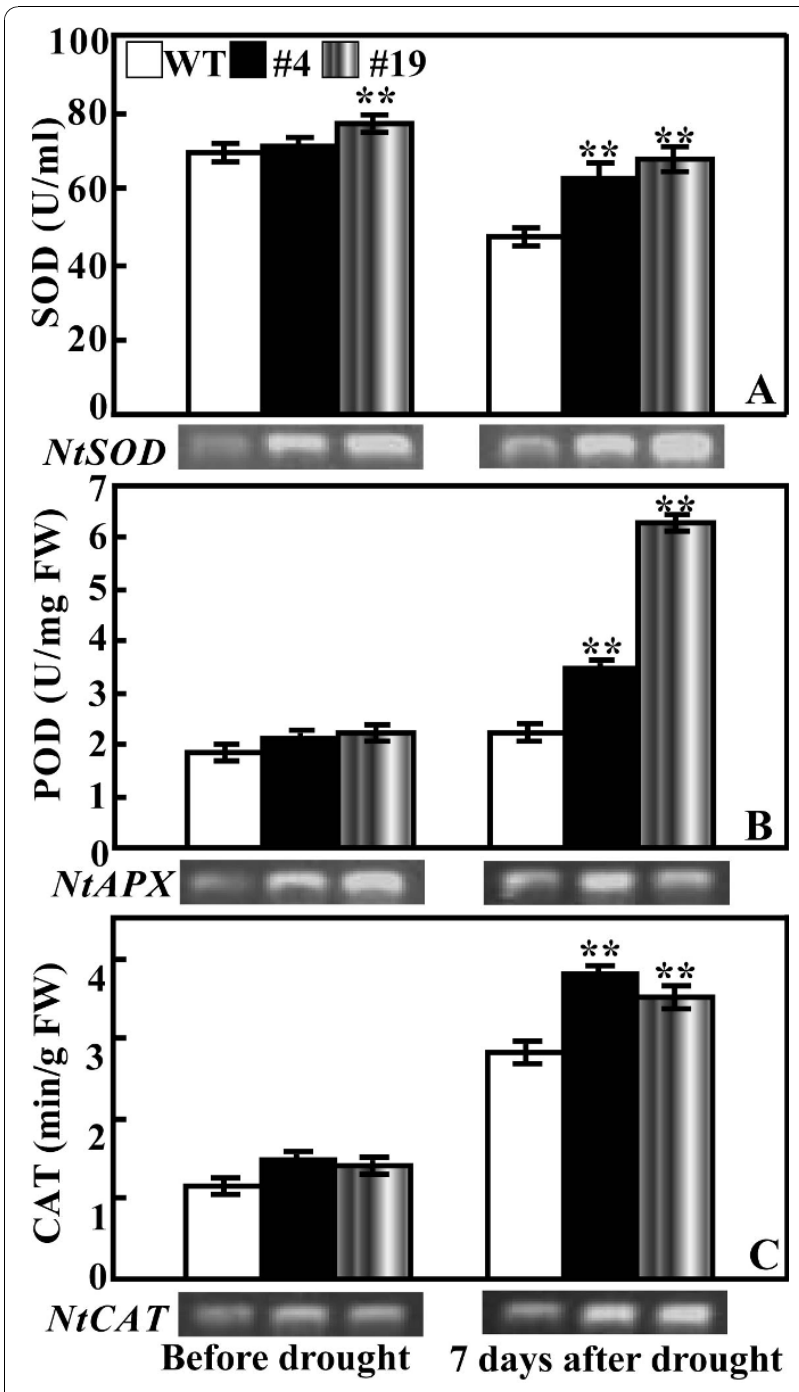

Figure 9 Activity and expression of antioxidant enzymes (genes) in wild type (WT), transgenic lines (\#4 and \#19) before and after drought treatment. (A-C) Activity of SOD (A), POD (B) and CAT (C) of WT, \#4 and \#19 plants before and after 7-d of drought treatment. Enzymes were extracted and assayed as described in 'Materials and methods'. ** indicates that the values of the two transgenic lines are significantly different those of WT $(P<$ 0.01). Expression of the genes encoding the enzymes was examined by RT-PCR and was shown below each graph.

upregulation of all nine genes in WT and the transgenic plants. However, it is noticeable that steady state mRNA levels of the genes in the two transgenic lines were still higher than WT, particularly NtADC2, NtLEA5, $N t E R D 10 C$ and NtDREB. These data indicated that overexpression of PtrABF in tobacco led to change in the transcript levels of endogenous stress-related genes.

\section{Discussion}

It has been well documented that upon exposure to abiotic stresses transcript levels of a multitude of genes 


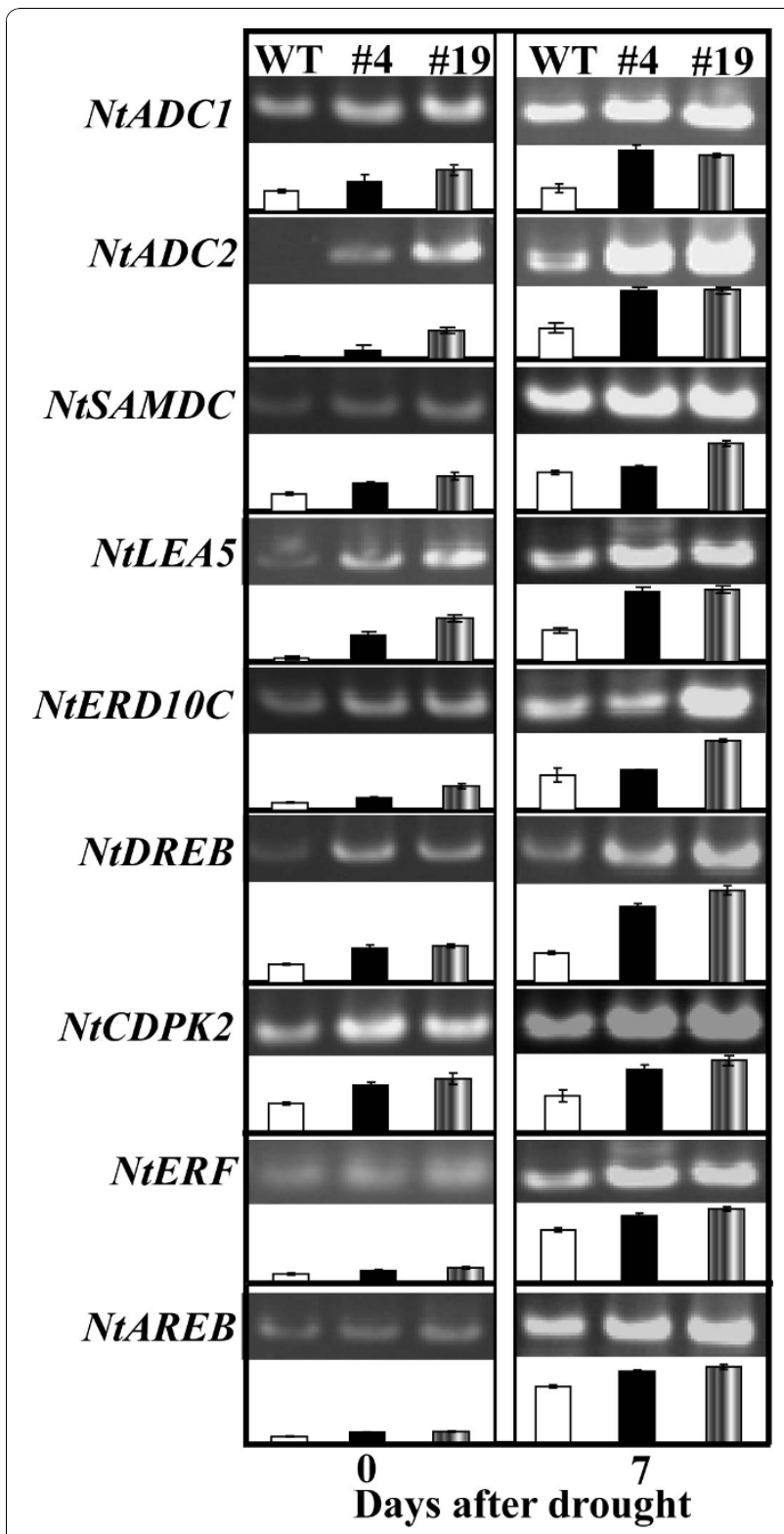

Figure 10 Expression profiles of the nine stress-responsive genes in wild type (WT), transgenic lines (\#4 and \#19) before and after drought treatment. RNA was extracted from leaves sampled at the onset and after $7 \mathrm{~d}$ of water stress, and reversetranscribed to synthesize CDNA, which was used for RT- PCR analysis with primers specific for these genes. mRNA levels of these genes were normalized to the transcripts of Tubulin in the same samples. The graph below each gel panel was made based on the means of three independent experiments that was calculated relative to the level of WT of $d 0$, taken as 1 .

are altered. Numerous studies have shown that ABFs/ AREBs play significant roles in regulating these stressrelated genes via interaction with ABRE cis-element in their promoters, suggesting that ABFs/AREBs are tightly involved in plant response to various adverse environment conditions. In this work, a gene encoding $\mathrm{ABF}$ from $P$. trifoliata was isolated by RT-PCR in combination with bioinformatics approach based on ESTs deposited in the public database. Sequence multiple alignment demonstrated that PtrABF showed high degree of sequence identity with ABFs of other plants retrieved from the database at amino acid level. A highly conserved bZIP domain consisting of a basic region responsible for DNA binding and three heptad leucine repeats related to TF dimerization [23] was observed near the C-terminus of PtrABF, indicating that PtrABF encoded a bZIP family protein. Apart from the bZIP domain, it also contained four highly conserved regions at the $\mathrm{N}$ or C-terminus (C1, C2, C3 and C4). Within these regions, several serine $(\mathrm{S})$ and threonine $(\mathrm{T})$ residues or consensus sequences are present, consistent with the structure of ABFs from other plants $[11,12]$. These conserved residues have been suggested as phosphorylation sites of different kinases, such as calmodulin-dependent protein kinase II (R/KXXS/T, position 28-31) and casein kinase II (S/TXXD/E, position 36-39), cGMP-dependent protein kinase (K/RXXXS/T, position 49-53), implying that activation of PtrABF might be regulated by protein phosphorylation, as has been reported in other AREBs/ ABFs $[12,15,24,25]$. Presence of these common characteristics demonstrated that PtrABF cloned in our study shared striking sequence similarity with other ABFs and may have a biological function same as or similar to them in abiotic stress response. The phylogenetic tree revealed relationship between PtrABF and ABFs from other plants, in which PtrABF was closely related to ABFs from dicots, including the ABFs of Arabidopsis thaliana. Although most ABFs in the tree remain to be characterized, in planta functions of ABFs of Arabidopsis have been determined, in which ABF3 and ABF4 act as activators of $A B A /$ stress response. In addition, overexpression of ABF2, ABF3 has been shown to affect multiple stress tolerance, implying that PtrABF might function in the same way as ABFs of Arabidopsis.

An important feature of plant ABFs is the induction of their transcript levels by abiotic stresses $[8,11,12,16]$. QRT-PCR analysis demonstrated that steady state mRNA levels of PtrABF were induced by ABA, dehydration and low temperature. Expression patterns of PtrABF were largely similar to ABF4/AREB2 that has been shown to be induced by ABA, salt, cold and drought [11]. However, it has to be mentioned that PtrABF was not induced by salt, different from ABF4. In Arabiopsis it has been shown that although ABFs were all induced by ABA, they are differentially regulated by various stresses and have been suggested to play various roles in stress response [11]. ABF family members might have specificity in their role under different stresses apart from existence of function redundancy, in which $\mathrm{ABF} 3$ and ABF4 played essential roles in germination 
control and $\mathrm{ABA}$ /stress response, whereas ABF2 was more closely implicated in seedling growth regulation and glucose response $[8,13]$. Induction of PtrABF by both dehydration and low temperature seems to indicate that this gene may participate in response to these cues.

Compared with low temperature, dehydration caused more profound induction of PtrABF mRNA abundance, which compelled us to do in-depth work on elucidation of the potential role of this gene for enhancing dehydration and drought tolerance. To this end, transgenic tobacco plants were produced via Agrobacteriummediated transformation of PtrABF under the control of CaMV 35S promoter. The two selected transgenic lines exhibited better phenotypic morphology, concomitant with less water loss, lower electrolyte leakage and higher chlorophyll content than wild type under either dehydration or long-term water stress, suggesting that overexpression of PtrABF conspicuously conferred tolerance to these adverse conditions. Apart from the water stress used herein, the transgenic plants also exhibited enhanced tolerance to low temperature treatment in comparison with WT (data not shown). Our work agreed with earlier reports, in which overexpression of ABF family members has been shown to render tolerance to multiple stresses in the same transgenic line [13,19-21,26], implying that ABFs class transcription factors hold great potential for genetically manipulating stress tolerance.

Despite the fact transformation of $A B F$ genes led to improvement of abiotic stress tolerance, the physiological mechanism underling the tolerance remained largely unknown. This stimulated us to carry out more work to find out physiological difference between the transgenic plants and WT under stress. We put special emphasis on comparing their ROS levels because it has been well accepted that in biological systems ROS accumulation is related to physiological perturbation and ROS levels can reflect the degree of damage to cellular components [27]. Histochemical staining by DAB and NBT clearly demonstrated that under dehydration and drought conditions the two transgenic lines accumulated remarkably less $\mathrm{O}_{2}$ - and $\mathrm{H}_{2} \mathrm{O}_{2}$ than WT. As ROS level during stresses greatly relies on the homeostasis between generation and removal [27], accumulation of less ROS in the transgenic lines seems to indicate that scavenging systems in these plants might work more effectively compared with WT. In order to detoxify stress-induced ROS, plants evolve a complex antioxidant system, in which several enzymes play essential roles, leading to scavenging ROS and protecting the cells against oxidative stress $[27,28]$. Of the enzymes, SOD provides the first line of defense against ROS by catalyzing the dismutation of $\mathrm{O}_{2}$ - to oxygen and $\mathrm{H}_{2} \mathrm{O}_{2}$, which was then scavenged by coordinated action of CAT and POD [29].
In our study, activities of SOD, POD and CAT in the two transgenic lines were not profoundly different from those of wild type under well-watering conditions although they were slightly higher in the transgenic lines, which sounds reasonable because under normal conditions ROS production remained at low levels and oxidative stress was not serious [27]. However, under water stress, activities of the three enzymes were significantly higher in the transgenic plants than WT, implying that the transgenic plants had more robust detoxifying system to eliminate ROS produced during stress, which is consistent with the dramatic reduction of ROS level and ROS-associated membrane damage (lower electrolyte leakage). It is noticed that activation of antioxidant enzymes was consistent with the upregulation of the three genes, suggesting that these enzymes may be regulated at transcriptional levels. Induction of genes involved in ROS scavenging has been previously reported when TF was ectopically expressed [30], suggesting that the TF might transcriptionally regulate the expression of genes related to oxidative reactions. Our work suggested that deployment of a better ROSscavenging system might be an integral part of defense against drought in the transgenic plants expressing PtrABF.

To cope with unfavorable environmental constraints plants modulate the expression of a large spectrum of stress-responsive genes, constituting an important molecular basis for the response and adaptation of plants to stresses $[22,31,32]$. In order to understand regulatory function of PtrABF and to explain the enhanced drought tolerance at molecular levels, transcript levels of nine stress-responsive genes were monitored before and after drought treatment, including five genes encoding functional proteins (NtADC1, NtADC2, NtSAMDC, $N t E R D 10 C$ and $N t L E A 5)$ and four encoding regulatory proteins (NtAREB, NtCDPK2, NtDREB and NtERF), which or whose homologues in other plants have been shown to be involved in abiotic stress response. RT-PCR analysis showed that steady-state mRNA levels of these genes were higher in the transgenic plants compared with those of WT in the absence of water stress, in line with earlier reports in which overexpression of a TF resulted in extensive alteration of transcript levels of an arsenal of related genes [33,34]. Although expression levels of all of the tested genes were upregulated by drought, they were still higher in the transgenic plants than in $\mathrm{WT}$, indicating that these genes were more intensely induced in the transgenic lines. NtADC1, $N t A D C 2$ and NtSAMDC are genes involved in biosynthesis of polyamines, which are low-molecular-weight polycations and have been shown to be important stress molecules $[35,36]$. Polyamines function in stress adaptation by acting as osmoticum regulator or membrane 
stabilizer through binding to macromolecules like proteins, nucleic acids and phospholipids of plasma membrane $[37,38]$. More drastic induction of these genes implied that the transgenic plants might synthesize higher levels of polyamines to prevent them from lethal injury and maintain better growth under water stress [39]. On the other hand, polyamines have been also proposed to act as free radical scavengers [40], and the larger induction of the polyamine biosynthetic genes agreed with lower ROS accumulation in the transgenic plants after drought stress. NtLEA5 and NtERD10C encode hydrophilic late embryogenesis abundant (LEA) proteins that are assumed to play critical roles in combating cellular dehydration [41]. Higher expression levels of these genes suggested that the transgenic plants might provide more chaperones for various substrates and maintain membrane integrity or efficiently bind water, which are important strategies for plants to sustain growth during drought $[41,42]$. Induction of these functional genes to higher levels suggested that the transgenic plants might synthesize more protective compounds (polyamines) or proteins (LEA), which, along with others that were not identified herein, provided better adaptive or defensive niches against water stress, leading to alleviation of cellular damage when they were subjected to drought. Upregulation of the above genes suggests that they might be transcriptionally regulated by PtrABF through binding of the cis-element ABRE in their promoter. Although we could not provide evidence for this speculation, ABRE has been discovered in the promoter of most genes encoding LEA proteins [41] and in AtADC2 and AtSAMDC2 of Arabidopsis [43]. In addition to the functional genes, it is noted that the $N t C D P K 2$ and NtDREB were also induced to higher levels in the transgenic lines with or without drought stress, while $N t E R F$ and $N t A R E B$ were only slightly induced upon water stress. Induction of their mRNA to higher levels raised the possibility of interaction between them and PtrABF in order to orchestrate well-defined stress tolerance machinery that functions in protection of the plants against adverse environment. Interestingly, the expression patterns of $N t C D P K 2$ and $N t D R E B$ were largely consistent with those of the functional genes before and after water stress. NtCDPK2 and NtDREB are important regulatory molecules involved in signal transduction or transcriptional regulation during stress conditions $[4,5,44,45]$. These genes may act as the intermediates between PtrABF and the aforementioned functional genes. In this case, PtrABF might function to facilitate transcriptional upregulation of these endogenous regulatory genes, which in turn activated their downstream target genes, including those mentioned above. In the future, extra work is needed to decipher the connection between these genes so as to gain more insight into the molecular mechanisms underlying PtrABF function in water stress tolerance.

\section{Conclusions}

A class-A bZIP transcription factor encoding PtrABF has been successfully isolated from $P$. trifoliata, which was induced by dehydration, low temperature and ABA. PtrABF was confirmed as a transcription factor due to subcellular localization in the nucleus, presence of transactivation activity and binding with ABRE.

Overexpression of PtrABF in tobacco enhanced tolerance to dehydration shock and long term water stress (drought). The tolerance may be ascribed to more robust activation of ROS scavenging system and coordinated and timely activation of an array of stress-responsive genes at molecular level, leading to synthesis of a wide range of protective compounds and proteins. These strategies may act in cooperation to render a more protective line of defence against the water stress.

The results presented here demonstrate that overexpression of PtrABF significantly modified drought tolerance in the transgenic plants, indicating that PtrABF might be a candidate gene with potential application to enhance abiotic stress tolerance.

\section{Methods}

Plant materials and stress treatments

Eight-month-old plants grown in the seedling beds at National Center of Citrus Breeding, Huazhong Agricultural University, were used to isolate the gene and to examine the gene expression patterns under different treatment. Uniform and healthy shoots were excised from the plants and inserted in a flask containing distilled water, which were kept for $1 \mathrm{~d}$ in a growth chamber at $25^{\circ} \mathrm{C}$ with 16 -h light/8-h dark photoperiod $\left(45 \mu \mathrm{mol} \mathrm{m}^{-2} \mathrm{~s}^{-1}\right)$ before exposure to the following treatment. For dehydration treatment, the shoots were placed on filter papers $(90 \times 90 \mathrm{~mm})$ and allowed to dry for 0, 0.5, 1, 3 and $6 \mathrm{~h}$. Low-temperature stress was imposed by transferring the shoots to $4^{\circ} \mathrm{C}$ for 0 , 5, 24, 48 and $72 \mathrm{~h}$. For salt or ABA treatment, the shoots were transferred to beakers containing fresh distilled water added with either $200 \mathrm{mM} \mathrm{NaCl}$ or $100 \mu \mathrm{M} \mathrm{ABA}$, kept in the same growth chamber for designated time $(0,5,24,48$ and $72 \mathrm{~h}$ for salt, $0,1,2,3,4$ and $5 \mathrm{~d}$ for ABA). For each treatment, a minimum of 30 shoots were used, and leaves were sampled from three randomly selected shoots at each time point and mixed as a material pool. The leaves from all of the treatments were frozen immediately in liquid nitrogen and stored at $-80^{\circ} \mathrm{C}$ until use for RNA extraction.

\section{Cloning and bioinformatics analysis of PtrABF}

In silico search against HarvEST http://harvest.ucr.edu, used as a primary sequence data set, was performed to get sequences that can be merged into one contig. In 
order to amplify a full-length cDNA encoding ABF, total RNA was extracted with TRIZOL reagent from leaves sampled from the shoots dehydrated for $6 \mathrm{~h}$. After treatment with RNase-free DNase (Promega, Madison, WI, USA) to remove genomic DNA contamination, $1 \mu \mathrm{g}$ of total RNA was reversely transcribed into cDNA with First Strand cDNA Synthesis Kit (Toyobo, Osaka, Japan) following the manufacturer's instructions, which was then used for RT-PCR to amplify the gene. Unless otherwise stated, PCR reaction in this research consisted of $100 \mathrm{ng}$ cDNA, $1 \times$ reaction buffer, $2.5 \mathrm{mM} \mathrm{MgCl} 2,0.25$ mM dNTP, $1 \mathrm{U}$ of Taq DNA polymerase (Fermentas) and $0.5 \mu \mathrm{M}$ of each primer designed based on the assembled contig (GSP1, Table 1) in a total volume of $25 \mu \mathrm{l}$. The PCR programme consisted of a 5 -min incubation at $94^{\circ} \mathrm{C}$, followed by 35 cycles of $30 \mathrm{~s}$ at $94^{\circ} \mathrm{C}, 30 \mathrm{~s}$ at $60^{\circ} \mathrm{C}$, and $90 \mathrm{~s}$ at $72^{\circ} \mathrm{C}$, followed by a 15 -min extension at $72^{\circ} \mathrm{C}$. The PCR product was sub-cloned into the pMD18$\mathrm{T}$ vector (TaKaRa, Dalian, China) to get pMD18-TPtrABF and sequenced (UnitedGene, Shanghai, China). cDNA and protein sequences were analyzed using the BLAST algorithm, and multiple alignments of the deduced amino acid sequence were performed using the ClustalW program. A phylogenetic relationship tree was constructed by the neighbor-joining (NJ) method using MEGA (version 4.0). Theoretical isoelectric point (pI) and molecular weight were also identified by tools in an internet server, ExPASy (Expert Protein Analysis System, http://www.expasy.org/tools). Prediction of the bZIP domain was performed on SMART (Simple Modular Architecture Research Tool, http://smart.embl-heidelberg.de/), a web-based protein database.

\section{Analysis of the gene expression by quantitative real-time PCR (QRT-PCR)}

QRT-PCR was performed according to [46] in order to evaluate expression levels of PtrABF under different treatment. RNA isolation and cDNA synthesis of the collected samples were performed as mentioned above. Each $10 \mu \mathrm{l}$ of PCR reaction contained $5 \mu \mathrm{l} 2 \times$ SYBR Green RealMasterMix (Applied Biosystems), $50 \mathrm{ng}$ cDNA, and $0.4 \mu \mathrm{M}$ of each primer specific for PtrABF (GSP2, Table 1), while Actin gene (Table 1) was used as internal control for expression normalization. QRT-PCR was performed in an ABI 7500 Real Time System (PE Applied Biosystems, Foster City, CA, USA) with following PCR program, $50^{\circ} \mathrm{C}$ for $2 \mathrm{~min}, 95^{\circ} \mathrm{C}$ for $10 \mathrm{~min}$, followed by 40 cycles of $95^{\circ} \mathrm{C}$ for $15 \mathrm{~s}$, and 57 (GSP2)/58 (Actin) ${ }^{\circ} \mathrm{C}$ for $1 \mathrm{~min}$. Each sample was amplified in four independent replicates.

\section{Subcellular localization of PtrABF}

The full-length ORF of PtrABF was amplified by PCR from pMD18-T-PtrABF with primers containing either
BamHI or XhoI restriction site (GSP3, Table 1), and the amplicon was ligated into $\mathrm{pMD} 18-\mathrm{T}$ to get $\mathrm{pMD} 18-\mathrm{T}_{\mathrm{B} /}$ $\mathrm{x}$-PtrABF. The GFP (AAB47998) coding region was PCR amplified from the pMD18-T vector with BamHI-KpnI linker primers (GFP-GSP1, Table 1), and cloned into the BamHI and KpnI sites of $\mathrm{pMD} 18-\mathrm{T}_{\mathrm{B} / \mathrm{X}-P \operatorname{trABF}}$, generating a recombinant construct of PtrABF-GFP, which was then inserted into the $X h o \mathrm{I}$ and $K p n \mathrm{I}$ cloning sites of a pBI121 vector (a kind gift of Dr. Junhong Zhang) to produce a fusion construct (pBI121-PtrABF-GFP) under the control of the constitutive $35 \mathrm{~S}$ promoter. After verification by sequencing, the fusion construct and control vector (pBI121-GFP, provided by Dr. Junhong Zhang) were introduced into Agrobacterium tumefaciens strain EHA105 by heat shock. Transformation of onion epidermal cells was done based on a previous method [47] and the onion cells were cultured on MS medium [48] for 2 $\mathrm{d}$, followed by observation of transient expression of GFP with a universal fluorescence microscope (Olympus BX61).

\section{Transcriptional activation and ABRE binding assay of PtrABF in yeast}

The transactivation experiment was carried out according to the manual of Matchmaker ${ }^{\text {ru }}$ Two-Hybrid System 3 (Clotech, USA). PCR amplification of pMD18-TPtrABF was carried out with a pair of specific primers (GSP4, in which BamHI and NcoI restriction sites were introduced), and the PCR product was double digested by $N c o I$ and $B a m H I$. The resultant fragment containing PtrABF $\left(\operatorname{PtrABF}_{\mathrm{N} / \mathrm{B}}\right)$ was purified and subcloned into downstream of the GAL4 DNA-binding domain of vector pGBKT7 linearized with $\mathrm{NcoI}$ and $\mathrm{BamHI}$ to get a fusion construct (pGBKT7-PtrABF). This construct and pGBKT7, used as a negative control, were separately transformed into Saccharomyces cerevisiae strain AH109 according to the manufacturer's instructions (Clontech). The transformants were streaked on the SD/-Trp or $\mathrm{SD} /$-Ade/-His/-Leu/-Trp medium supplemented with different concentration of 3-AT $(0,5$ and $15 \mathrm{mM})$ and incubated at $30^{\circ} \mathrm{C}$ for $4 \mathrm{~d}$, followed by evaluation of growth status of the transformants. In addition, the cells were also cultured on SD/-Ade/-His/-Leu medium added with both $15 \mathrm{mM} 3-\mathrm{AT}$ and $20 \mathrm{mM} \mathrm{X- \alpha -Gal} \mathrm{for}$ examination of $\beta$-galactosidase activity (Clontech).

A yeast one-hybrid assay (Clontech, Palo Alto, CA) was used to investigate if PtrABF bound to ABRE. For this purpose, $P \operatorname{tr} A B F_{N / B}$ mentioned above was fused to the GAL4 activation domain in the vector pGADT7 digested with $\mathrm{BamHI}$ and NcoI to get pGAD-PtrABF. A 42-bp oligonucleotide sequence containing three tandem repeat copies of 5'-GGACACGTGGCG-3' (ABRE is underlined) and restriction sites of $E c o R I$ and $S a c I$ was synthesized and cloned into multiple cloning sites upstream from the 
Table 1 Oligonucleotide primers used in this study

\begin{tabular}{|c|c|c|c|c|c|c|}
\hline \multirow[t]{2}{*}{ Genes } & \multirow[t]{2}{*}{ Primers } & \multirow{2}{*}{$\begin{array}{l}\text { Annealing } \\
\text { temperature }\left({ }^{\circ} \mathrm{C}\right)\end{array}$} & \multirow{2}{*}{$\begin{array}{l}\text { Product size } \\
\text { (bp) }\end{array}$} & \multicolumn{2}{|l|}{ Sequences $\left(5^{\prime}-3^{\prime}\right)$} & \multirow[b]{2}{*}{ Used for } \\
\hline & & & & Forward & Reverse & \\
\hline PtrABF & GSP1 & 60 & 1511 & ATTGGAGCAAGCTGTTGCCACCGC & CGCAGCTGCCTACACTCCATGG & $\begin{array}{l}\text { Amplification of full- } \\
\text { length cDNA }\end{array}$ \\
\hline Actin & Actin & 58 & 107 & ATTGTAAGCAACTGGGATGATA & AGAGGTGCCTCAGTGAGAAG & $\begin{array}{l}\text { Quantitative real-time } \\
\text { PCR }\end{array}$ \\
\hline PtrABF & GSP2 & 57 & 113 & CCATCACCAGTTCCTTATGTGTT & GATCTTGCAGCTGACTCTCTGTT & \\
\hline PtrABF & GSP3 & 58 & 1363 & $\begin{array}{l}\text { GGCTCGAGATGGGATCTCAAATGAACTTCAAG (Xhol } \\
\text { site is underlined) }\end{array}$ & $\begin{array}{l}\text { AAGGATCCCGTCAGTGTCCTCCTCAAGCACAG (BamHI } \\
\text { site is underlined) }\end{array}$ & Subcellular localization \\
\hline GFP & $\begin{array}{l}\text { GFP- } \\
\text { GSP1 }\end{array}$ & 60 & 732 & $\begin{array}{l}\text { CGGGATCCATGAGTAAAGGAGAAG } \\
\text { (BamHI site is underlined) }\end{array}$ & $\begin{array}{l}\text { CGGTACCTTATTTGTATAGTTCATCC (Kpnl site is } \\
\text { underlined) }\end{array}$ & \\
\hline PtrABF & GSP4 & 60 & 1366 & $\begin{array}{l}\text { GGCCATGGCGATGGGATCTCAAATGAACTTCAAG (Ncol } \\
\text { site is underlined) }\end{array}$ & $\begin{array}{l}\text { ATAGGATCCCTACCAAGGGCCCGTCAGTGTCC (BamHI } \\
\text { site is underlined) }\end{array}$ & $\begin{array}{l}\text { Transcriptional activation } \\
\text { assay }\end{array}$ \\
\hline PtrABF & GSP5 & 58 & 160 & ATTGGAGCAAGCTGTTGCCACCGC & CGCAGCTGCCTACACTCCATGG & $\begin{array}{l}\text { Transgene } \\
\text { overexpression }\end{array}$ \\
\hline $\begin{array}{l}\text { CaMV35S- } \\
\text { PtrABF }\end{array}$ & $\begin{array}{l}35 S- \\
\text { PtrABF }\end{array}$ & 60 & 2013 & CGCCGTAAAGACTGGCGAACAGTTCATACAGAGT & CGCAGCTGCCTACACTCCATGG & $\begin{array}{l}\text { Confirmation of } \\
\text { transgenic plants }\end{array}$ \\
\hline NPTII & NPTII & 56 & 742 & AGACAATCGGCTGCTCTGAT & TCATTTCGAACCCCAGAGTC & \\
\hline NtADC1 & & 58 & 306 & CTTGCTGATTACCGCAATTTATC & CCTTACTGCAGGCTTTTCATCTA & $\begin{array}{l}\text { Analysis of expression } \\
\text { levels }\end{array}$ \\
\hline NtADC2 & & 58 & 350 & GCCGGCCCTAGGTTGTTGTGTAGATG & AGCGAACAACAAGAGGCAGCTGAAGCC & \\
\hline NtAREB & & 58 & 224 & TCTTCACAGCAAAAGCCTCA & GTGACCCCATTATGCAATCC & \\
\hline NtCAT & & 58 & 151 & AGGTACCGCTCATTCACACC & AAGCAAGCTTTTGACCCAGA & \\
\hline NtCDPK2 & & 58 & 267 & AGGTGAGCTTTTCGATAGGATTATT & ACTTCTGGTGCAACATAGTAAGGAC & \\
\hline NtDREB & & 58 & 503 & GGACCCACTTGCTGATTCTT & GCGCCTCCTCATCCATATAA & \\
\hline NtERD10C & & 58 & 366 & ACGTGGAGGCTACAGATCGTGGTTTG & TCTCCACTGGTACAGCCGTGTCCTCAC & \\
\hline NtERF & & 58 & 220 & ACACTTCATTTTCACATTCCAACTT & TCTTCTAATTCTTGACCATGGCTAC & \\
\hline NtLEA5 & & 58 & 350 & TTGTTAGCAGGCGTGGGTAT & CTCTCGCTCTTGTTGGGTTC & \\
\hline NtAPX & & 58 & 262 & CAAATGTAAGAGGAAACTCAGAGGA & AGCAACAACTCCAGCTAATTGATAG & \\
\hline NtSAMDC & & 58 & 313 & ATTGGTTTGAAGGTTITGAGAAG & TCACGTCTTGTACTTTGAGAGACAG & \\
\hline NtSOD & & 58 & 238 & AGCTACATGACGCCATTTCC & CCCTGTAAAGCAGCACCTTC & \\
\hline Tubulin & & 58 & 163 & TCCAGGACAAGGAGGGTAT & CATCAACAACAGGCAACCTAG & \\
\hline
\end{tabular}


HIS3 minimal promoter in the pHIS2 expression vector that had been digested with EcoRI and SacI to get the reporter vector $\mathrm{pHIS2-ABRE.} \mathrm{Both} \mathrm{pGADT7-PtrABF} \mathrm{and}$ pHIS2-ABRE were co-transformed into yeast strain Y187, while pGADT7/pHIS2-ABRE and pGADT7-PtrABF/ pHIS2 were also co-transformed as negative controls. The cells were cultured on SD/-His/-Leu/-Trp plates with or without 3-AT to identify the DNA-protein interaction.

\section{Transformation of tobacco and regeneration of transgenic plants}

pMD18-T-PtrABF was restricted with SalI and KpnI, and the resulting product was inserted into XhoI-KpnI sites of the binary vector $\mathrm{pBI} 121$ to generate $\mathrm{pBI} 121$ PtrABF, which was introduced into A. tumefaciens strain EHA 105 by heat shock after fidelity verification by sequencing. Seeds of Nicotiana nudicaulis were sterilized for $20 \mathrm{~s}$ in $70 \%(\mathrm{v} / \mathrm{v})$ ethanol and incubated in $2.5 \%(\mathrm{v} / \mathrm{v}) \mathrm{H}_{2} \mathrm{O}_{2}$ for $7 \mathrm{~min}$, followed by rinse with sterile distilled water for four times before they were sown on germination medium (GM) containing MS salts, $30 \mathrm{~g} \mathrm{l}^{-1}$ sucrose and $0.75 \%$ agar (pH 5.7). Seventy-day-old seedlings were used for transformation based on a leaf disc transformation method [49]. The infected explants were co-cultivated on GM added with $2.0 \mathrm{mg} \mathrm{l}^{-1} 6$-BA and $0.3 \mathrm{mg} \mathrm{l}^{-1} \mathrm{NAA}$ for $3 \mathrm{~d}$ at $25^{\circ} \mathrm{C}$ in the dark, and then transferred on selection medium (GM, $2.0 \mathrm{mg} \mathrm{l}^{-1} 6-\mathrm{BA}$, $0.3 \mathrm{mg} \mathrm{l}^{-1} \mathrm{NAA}, 50 \mathrm{mg} \mathrm{l}^{-1}$ kanamycin and $400 \mathrm{mg} \mathrm{l}^{-1}$ cefotaxime). After selection for $30 \mathrm{~d}$, the kanamycinresistant shoots were shifted to fresh selection medium for further screening, and the resistant shoots were transferred to rooting medium (GM, $0.3 \mathrm{mg} \mathrm{l}^{-1} \mathrm{NAA}$, $50 \mathrm{mg} \mathrm{l}^{-1}$ kanamycin, $400 \mathrm{mg} \mathrm{l}^{-1}$ cefotaxime) to induce roots. Presence of the transgene in the kanamycinresistant seedlings was confirmed by PCR as described below. Putative transgenic T0 plants were maintained in a growth chamber with a $16 \mathrm{~h}$ light $/ 8 \mathrm{~h}$ dark photoperiod at $25^{\circ} \mathrm{C}$ until they flowered and set seeds to select homozygous lines [50].

\section{Confirmation of the transgenic plants by PCR}

Genomic DNA was extracted from T0 in vitro seedlings and wild type using a cetyltrimethyl ammonium bromide (CTAB)-based method. PCR reaction solution was the same as that of RT-PCR except the use of DNA template and primers specific to both NPTII gene and CaMV35S and PtrABF (Table 1). Amplifications were performed at $94^{\circ} \mathrm{C}$ for $5 \mathrm{~min}$, followed by 35 cycles of $94^{\circ} \mathrm{C}$ for $45 \mathrm{~s}, 60$ (35S-PtrABF) $/ 56$ (NPTII) ${ }^{\circ} \mathrm{C}$ for $45 \mathrm{~s}$, $72^{\circ} \mathrm{C}$ for $1-2 \mathrm{~min}$, and a final 15 -min extension at $72^{\circ} \mathrm{C}$. PCR products were electrophoresesed on $1.0 \%(\mathrm{w} / \mathrm{v})$ agarose gel containing $0.5 \mu \mathrm{g} \mathrm{l}^{-1}$ ethidium bromide and visualized under UV transillumination.

\section{Dehydration shock and drought tolerance of the transgenic plants}

Seeds of the transgenic lines and wild type were sterilized and sown on germination medium as mentioned above. Some of the in vitro seedlings were subjected to dehydration and the others were transplanted to soil pots in a growth chamber for drought treatment. For dehydration analysis, the aerial parts of 35-d-old in vitro seedlings of each line were put on clean filter papers and allowed to dry for up to $90 \mathrm{~min}$. Fresh weight of the samples was measured every $10 \mathrm{~min}$ to determine the rate of water loss relative to the initial value. Electrolyte leakage and accumulation of $\mathrm{O}_{2}$ - and $\mathrm{H}_{2} \mathrm{O}_{2}$ in the leaves at the last time point were examined as described below.

The seedlings used for drought treatment were kept on flat-bottom trays to grow for 4 months with regular irrigation prior to withholding water for $21 \mathrm{~d}$. Electrolyte leakage and total chlorophyll content of the plants exposed to drought for $7 \mathrm{~d}$ were measured, while leaves sampled at the onset of and $7 \mathrm{~d}$ after drought were frozen immediately in liquid nitrogen and stored at $-80^{\circ} \mathrm{C}$ until use for analysis of enzyme activity and gene expression. Accumulation of $\mathrm{O}_{2}-$ and $\mathrm{H}_{2} \mathrm{O}_{2}$ in the plants subjected to 21-d water stress was examined.

\section{Measurement of electrolyte leakage (EL) and total chlorophyll content}

EL was measured according to [51] with slight modification. The collected leaves were stripped and placed in $25 \mathrm{ml}$ distilled water, shaken on a gyratory shaker (200 $\mathrm{rpm}$ ) at room temperature for $2 \mathrm{~h}$, and the initial conductivity $(\mathrm{C} 1)$ was measured with a conductivity meter (DSS-307, Shanghai, China). The samples were then boiled for $10 \mathrm{~min}$ to induce maximum leakage. After cooling down at room temperature electrolyte conductivity $(\mathrm{C} 2)$ was measured, and the relative electrolyte leakage (C\%) was calculated based on $100 \times \mathrm{C} 1 / \mathrm{C} 2$.

Total chlorophyll was extracted and assayed as described previously [52]. About $0.1 \mathrm{~g}$ fine powder of leaf tissue was homogenized in $1 \mathrm{ml}$ of $80 \%$ acetone and kept for $15 \mathrm{~min}$ at room temperature in dark. The crude extraction was centrifuged for $20 \mathrm{~min}$ at $10000 i$ (room temperature), and the resultant supernatant was used for assessing absorbance at 663, 645 and $480 \mathrm{~nm}$ with a spectrophotometer (Shimadzu UV-1600, Japan), and total chlorophyll content was computed in terms of fresh weight (FW).

In situ histochemical localization of $\mathrm{O}_{2}-$ and $\mathrm{H}_{2} \mathrm{O}_{2}$

In situ accumulation of $\mathrm{O}_{2}$ - and $\mathrm{H}_{2} \mathrm{O}_{2}$ was examined based on histochemical staining by nitroblue tetrazolium (NBT) and 3, 3'-diaminobenzidine (DAB), respectively [39]. For $\mathrm{O}_{2}$ - detection, the leaves from both wild type 
and the transgenic plants were immersed in $1 \mathrm{mg} \mathrm{ml}^{-1}$ fresh NBT solution (prepared in $10 \mathrm{mM}$ phosphate buffer, $\mathrm{pH}$ 7.8) at ambient temperature until appearance of dark spots. For detection of $\mathrm{H}_{2} \mathrm{O}_{2}$, the leaves were inserted into $1 \mathrm{mg} \mathrm{ml}^{-1}$ fresh DAB solution ( $\mathrm{pH}$ 3.8) prepared in $10 \mathrm{mM}$ phosphate buffer $(\mathrm{pH} 7.8)$ and incubated in light until brown spots were observed. The stained leaves were then bleached in concentrated ethanol, and kept in $70 \%$ ethanol.

\section{Measurement of activity of antioxidant enzymes}

For extraction of peroxidase (POD, EC 1.11.1.7), catalase (CAT, EC 1.11.1.6) and superoxide dismutase (SOD, EC 1.15.1.1), about $0.5 \mathrm{~g}$ of leaf sample was ground in liquid nitrogen with pre-cooled pestle and mortar, and homogenized in $5 \mathrm{ml}$ of extraction buffer containing $50 \mathrm{mM}$ phosphate buffer (pH7.8) and 1\% polyvinylpyrrolidone. The homogenate was centrifuged at $10000 \mathrm{~g}$ for $20 \mathrm{~min}$ at $4{ }^{\circ} \mathrm{C}$ and the resulting supernatant was collected for enzyme activity analysis. Activities of SOD, expressed as unit (U) $\mathrm{ml}^{-1}$, were spectrophotometrically measured using SOD Detection Kit (A001, Jiancheng, Nanjing, China) according to the manufacturer's instruction. POD activity, expressed as $\mathrm{U} \mathrm{mg}^{-1} \mathrm{FW}$, was assayed according to [39] with slight modification. The assay mixture in a final volume of $3.0 \mathrm{ml}$, which contained 1 $\mathrm{ml}$ of $0.05 \mathrm{M}$ phosphate buffer ( $\mathrm{pH} 7.0), 1 \mathrm{ml}$ of $0.3 \%$ $\mathrm{H}_{2} \mathrm{O}_{2}, 0.95 \mathrm{ml}$ of $0.2 \%$ guaiacol and $50 \mu \mathrm{l}$ of enzyme extract, was incubated for $3 \mathrm{~min}$ at $34^{\circ} \mathrm{C}$. Activity of POD was determined based on the increase in absorbance read at $470 \mathrm{~nm}$, and one unit of POD activity was defined as the increase of absorbance by 0.01 per min. CAT activity was measured by the depletion of $\mathrm{H}_{2} \mathrm{O}_{2}$ at $240 \mathrm{~nm}$ [53]. The sample solution was composed of $0.1 \% \mathrm{H}_{2} \mathrm{O}_{2}, 100 \mathrm{mM}$ phosphate buffer ( $\mathrm{pH} 7.0$ ) and 100 $\mu \mathrm{l}$ enzyme extract in a total volume of $3 \mathrm{ml}$. The CAT activity, expressed as $\mathrm{U} \mathrm{g}^{-1} \mathrm{FW}$, was assessed by monitoring the decrease in absorbance at $240 \mathrm{~nm}$ as a consequence of $\mathrm{H}_{2} \mathrm{O}_{2}$ consumption, and one unit of CAT activity was defined as reduction of the absorbance by 0.01 per min.

Semi-quantitative RT-PCR for analysis of gene expression Semi-quantitative RT-PCR was utilized to examine overexpression of the transgene (PtrABF) in the transgenic lines, and to monitor transcriptional levels of nine stress-responsive genes, including NtADC1 (AF127239.1), NtADC2 (7230374), NtSAMDC (AB304782.1), NtLEA5 (AF053076), NtERD10C (AB049337.1), NtERF (AY655738.1), NtCDPK2 (AJ344156.1), NtDREB(EU727157.1), NtAREB (BAB61098), and three genes encoding the antioxidant enzymes, NtCAT (U93244.1), NTAPX (U15933.1, as gene coding for POD was not retrieved, it was used as a substitute) and NtSOD
(AB093097). Extraction of total RNA and cDNA synthesis of the samples collected before and after drought treatment were done as described for cloning of PtrABF. Genespecific primers for PtrABF (GSP5, Table 1) and the stressrelated genes were designed by Primer 5.0 based on the public sequences (Table 1). RT-PCR amplification of each gene was performed using $100 \mathrm{ng}$ of cDNA as a template and two corresponding specific primers with the following program, an initial $5 \mathrm{~min}$ denaturing at $94^{\circ} \mathrm{C}, 31$ cycles of $30 \mathrm{~s}$ at $94^{\circ} \mathrm{C}, 40 \mathrm{~s}$ at $58^{\circ} \mathrm{C}, 40 \mathrm{~s}$ at $72^{\circ} \mathrm{C}$, and a 10 -min extension at $72^{\circ} \mathrm{C}$. As an internal control to show that equal amounts of total RNA has been loaded, the cDNAs were also amplified with a pair of tobacco Tubulin gene primers (Table 1) with the same procedure. Detection of amplification products followed the procedure for PCR of genomic DNA. RT-PCR was repeated three times to ensure reliability of the data. In order to compare the expression difference quantitatively, expression level of the nine stress-responsive gene was normalized to the Tubulin gene by densitometric quantitative analysis using a software (Quantity One 4.6.2). Expression level of WT at the onset of water stress was set to 1 , and those of the other samples were quantified accordingly.

\section{Statistical analysis}

Dehydration and drought treatment of the transgenic lines and WT were repeated at least twice with three replicates for each line with consistent results, and the results of a representative experiment were presented, shown as mean $\pm \mathrm{SE}$. The data were analyzed using analysis of variance (ANOVA) by SAS software (version 8.0, SAS Institute, NC, USA), and statistical difference was compared based on Fisher's LSD test.

\section{Acknowledgements}

This work was supported by National Natural Science Foundation of China (30623012, 30871685, 30921002, 31071778), the Research Fund for the Doctoral Program of Higher Education (20090146110010), Fok Ying Tong Education Foundation (114034), Yunnan Provincial Tobacco Corporation (07A04) Hubei Provincial Natural Science Foundation (2009CDA080) and the National High Technology Research and Development Program (863 Program) of China. The authors are grateful to Mr. Tao Luo for his help with transactivation analysis.

\section{Author details}

${ }^{1}$ National Key Laboratory of Crop Genetic Improvement, Key Laboratory of Horticultural Plant Biology of Ministry of Education, Huazhong Agricultural University, Wuhan 430070 China. ${ }^{2}$ Yunnan Academy of Tobacco Agricultural Sciences, Yuxi 653100, China.

\section{Authors' contributions}

$\mathrm{XSH}$ and $\mathrm{JHL}$ designed experiments and XJC provides the tobacco seeds. $\mathrm{XSH}$ performed the experimental procedures and drafted the manuscript. $J H L$ revised critically the manuscript and finalized it. All of the authors read and approved the final manuscript.

Received: 8 June 2010 Accepted: 25 October 2010 Published: 25 October 2010 


\section{References}

1. Faroog M, Wahid A, Kobayashi N, Fujita D, Basra SMA: Plant drought stress: effects, mechanisms and management. Agron Sustain Dev 2009, 29:185-212.

2. Seki M, Narusaka M, Abe H, Kasuga M, Yamaguchi-Shinozaki K, Carninci $P$, Hayashizaki $Y$, Shinozaki K: Monitoring the expression pattern of 1300 Arabidopsis genes under drought and cold stresses by using a fulllength cDNA microarray. Plant Cell 2001, 13:61-72.

3. Seki M, Narusaka M, Ishida J, Nanjo T, Fujita M, Oono $Y$, AKamiya A Nakajima M, Enju A, Sakurai T, Satou M, Akiyama K, Taji T, Kazuko Yamaguchi-Shinozaki K, Carninci P, Kawai J, Hayashizaki Y, Shinozaki K: Monitoring the expression profiles of 7000 Arabidopsis genes under drought, cold, and high-salinity stresses using a full-length cDNA microarray. Plant J 2002, 31:279-292.

4. Yamaguchi-Shinozaki $K$, Shinozaki $K$ : Transcriptional regulatory networks in cellular responses and tolerance to dehydration and cold stresses. Annu Rev Plant Biol 2006, 57:781-803.

5. Shinozaki K, Yamaguchi-Shinozaki K: Gene networks involved in drought stress response and tolerance. J Exp Bot 2007, 58:221-227.

6. Zhu JK: Salt and drought stress signal transduction in plants. Annu Rev Plant Physiol 2002, 53:247-273.

7. Nakashima K, Ito Y, Yamaguchi-Shinozaki K: Transcriptional regulatory networks in response to abiotic stresses in Arabidopsis and grasses. Plant Physiol 2009, 149:88-95.

8. Kim SY: The role of ABF family bZIP class transcription factors in stress response. Physiol Plant 2006, 126:519-527.

9. Hurst HC: Transcription factors 1. bZIP proteins. Protein Profile 1994, 1:123-168.

10. Jakoby M, Weisshaar B, Droge-Laser W, Vicente-Carbajosa J, Tiedemann J, Kroj T, Parcy F: bZIP transcription factors in Arabidopsis. Trends Plant Sci 2002, 7:106-111.

11. Choi $H$, Hong JH, Ha JO, Kang JY, Kim SY: ABFs, a family of ABAresponsive element binding factors. J Biol Chem 2000, 275:1723-1730.

12. Uno $Y$, Furuhata $T$, Abe $H$, Yoshida $R$, Shinozaki $K$, Yamaguchi-Shinozaki K: Arabidopsis basic leucine zipper transcription factors involved in an abscisic acid- dependent signal transduction pathway under drought and high-salinity conditions. Proc Natl Acad Sci USA 2000, 97:11632-11637.

13. Kim S, Kang JY, Cho DI, Park JH, Kim SY: ABF2, an ABRE-binding bZIP factor, is an essential component of glucose signaling and its overexpression affects multiple stress tolerance. Plant J 2004, 40:75-87.

14. Fujita Y, Fujita M, Satoh R, Maruyama K, Parvez MM, Seki M, Hiratsu K, Ohme-Takagi M, Shinozaki K, Yamaguchi-Shinozaki K: AREB1 is a transcription activator of novel ABRE-dependent $A B A$ signaling that enhances drought stress tolerance in Arabidopsis. Plant Cell 2005, 17:3470-3488.

15. Yoshida T, Fujita Y, Sayama H, Kidokoro S, Maruyama K, Mizoi J, Shinozaki K, Yamaguchi-Shinozaki K: AREB1, AREB2, and ABF3 are master transcription factors that cooperatively regulate $A B R E$-dependent $A B A$ signaling involved in drought stress tolerance and require $A B A$ for full activation. Plant J 2010, 61:672-685.

16. Yáñez M, Cáceres S, Orellana S, Bastías A, Verdugo I, Ruiz-Lara S, Casaretto JA: An abiotic stress-responsive bZIP transcription factor from wild and cultivated tomatoes regulates stress-related genes. Plant Cell Rep 2009, 28:1497-1507.

17. Hossain MA, Lee Y, Cho Jl, Ahn CH, Lee SK, Jeon JS, Kang H, Lee CH, An G, Park PB: The bZIP transcription factor OsABF1 is an ABA responsive element binding factor that enhances abiotic stress signaling in rice. Plant Mol Biol 2010, 72:557-566

18. Kang JY, Choi HI, Im MY, Kim SY: Arabidopsis basic leucine zipper proteins that mediate stress-responsive abscisic acid signaling. Plant Cell 2002, 14:343-357.

19. Kim JB, Kang JY, Kim SY: Over-expression of a transcription factor

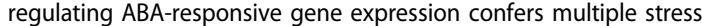
tolerance. Plant Biotechnol J 2004, 2:459-466.

20. Vanjildorj E, Bae TW, Riu KZ, Kim SY, Lee HY: Overexpression of Arabidopsis ABF3 gene enhances tolerance to drought and cold in transgenic lettuce (Lactuca sativa). Plant Cell Tiss Org Cult 2005, 83:41-50.

21. Vanjildorj E, Bae TW, Riu KZ, Yun PY, Park SY, Lee CH, Kim SY, Lee HY: Transgenic Agrostis mongolica Roshev. with enhanced tolerance to drought and heat stresses obtained from Agrobacterium-mediated transformation. Plant Cell Tiss Org Cult 2006, 87:109-120.
22. Umezawa T, Fujita M, Fujita Y, Yamaguchi-Shinozaki K, Shinozaki K: Engineering drought tolerance in plants: discovering and tailoring genes to unlock the future. Curr Opin Biotech 2006, 17:113-122.

23. Corrêa LGG, Riaño-Pachón DM, Schrago CG, Vicentini dos Santos R, MuellerRoeber $B$, Vincentz M: The role of bZIP transcription factors in green plant evolution: adaptive features emerging from four founder genes. PLOS ONE 2008, 3:e2944.

24. Kobayashi $Y$, Murata M, Minami H, Yamamoto S, Kagaya Y, Hobo T, Yamamoto A, Hattori T: Abscisic acid-activated SNRK2 protein kinases function in the gene-regulation pathway of $A B A$ signal transduction by phosphorylating ABA-response element-binding factors. Plant J 2005, 44:939-949.

25. Furihata T, Maruyama K, Fujita Y, Umezawa T, Yoshida R, Shinozaki K, Yamaguchi-Shinozaki K: Abscisic acid dependent multisite phosphorylation regulates the activity of a transcription activator AREB1. Proc Natl Acad Sci USA 2006, 103:1988-1993.

26. Oh SJ, Song SI, Kim YS, Jang HJ, Kim SY, Kim M, Kim YK, Nahm BH, Kim JK Arabidopsis CBF3/DREB1A and ABF3 in transgenic rice increased tolerance to abiotic stress without stunting growth. Plant Physiol 2005 138:341-351.

27. Miller G, Suzuki N, Ciftci-Yilmaz S, Mittler R: Reactive oxygen species homeostasis and signaling during drought and salinity stresses. Plant Cell Environ 2010, 33:453-457.

28. Jaleel CA, Riadh K, Gopi R, Manivannan P, Inès J, Al-Juburi HJ, Zhao CX, Shao HB, Panneerselvam R: Antioxidant defense response: physiological plasticity in higher plants under abiotic constraints. Acta Physiol Plant 2009, 31:427-436.

29. Blokhina O, Virolainen E, Fagerstedt KV: Antioxidants, oxidative damage and oxygen deprivation stress: a review. Ann Bot 2003, 91:179-194.

30. Wu L, Zhang Z, Zhang H, Wang XC, Huang R: Transcriptional modulation of ethylene response factor protein JERF3 in the oxidative stress response enhances tolerance of tobacco seedlings to salt, drought, and freezing. Plant Physiol 2008, 148:1953-1963.

31. Chinnusamy V, Zhu J, Zhu JK: Cold stress regulation of gene expression in plants. Trends Plant Sci 2007, 12:444-451.

32. Hirayama T, Shinozaki K: Research on plant abiotic stress responses in the post-genome era: past, present and future. Plant J 2010, 61:1041-1052.

33. Vogel JT, Zarka DG, Van Buskirk HA, Fowler SG, Thomashow MF: Roles of the CBF2 and ZAT12 transcription factors in configuring the low temperature transcriptome of Arabidopsis. Plant J 2005, 41:195-211.

34. Dai X, Xu Y, Ma Q, Xu W, Wang T, Xue Y, Chong K: Overexpression of an R1R2R3 MYB gene, OsMYB3R-2, increases tolerance to freezing, drought, and salt stress in transgenic Arabidopsis. Plant Physiol 2007, 143:1739-1751

35. Rhee HJ, Kim EJ, Lee JK: Physiological polyamines: simple primordial stress molecules. J Cell Mol Med 2007, 11:685-703.

36. Liu JH, Kitashiba H, Wang J, Ban Y, Moriguchi T: Polyamines and their ability to provide environmental stress tolerance to plants. Plant Biotechnol 2007, 24:117-126.

37. Evans PT, Malmberg RL: Do polyamines have roles in plant development? Annu Rev Plant Physiol Plant Mol Biol 1989, 40:235-269.

38. Aziz A, Larher F: Changes in polyamine titers associated with the proline response and osmotic adjustment of rape leaf discs submitted to osmotic stresses. Plant Sci 1995, 112:175-186.

39. Shi J, Fu XZ, Peng T, Huang XS, Fan QJ, Liu JH: Spermine pretreatment confers dehydration tolerance of citrus in vitro plants via modulation of antioxidative capacity and stomatal response. Tree Physiol 2010, 30:914-922.

40. Tadolini B: Polyamine inhibition of lipoperoxidation. The influence of polyamines on iron oxidation in the presence of compounds mimicking phospholipid polar heads. Biochem J 1988, 249:33-36.

41. Hundertmark $M$, Kincha DK: LEA (Late Embryogenesis Abundant) proteins and their encoding genes in Arabidopsis thaliana. BMC Genomics 2008, 9:118

42. Kovacs D, Kalmar E, Torok Z, Tompa P: Chaperone activity of ERD10 and ERD14, two disordered stress-related plant proteins. Plant Physiol 2008, 147:381-390.

43. Urano K, Yoshiba $Y$, Nanjo T, Igarashi $Y$, Seki M, Sekiguchi F, YamaguchiShinozaki K, Shinozaki K: Characterization of Arabidopsis genes involved in biosynthesis of polyamines in abiotic stress response and developmental stages. Plant Cell Environ 2003, 26:1917-1926. 
44. Witte $C P$, Keinath $N$, Dubiella U, Demoulière $R$, Seal $A$, Romeis $T$ : Tobacco calcium-dependent protein kinases are differentially phosphorylated in vivo as part of a kinase cascade that regulates stress response. J Biol Chem 2010, 285:9740-9748.

45. Saibo NJM, Lourenço T, Oliveira MM: Transcription factors and regulation of photosynthetic and related metabolism under environmental stresses. Ann Bot 2009, 103:609-623.

46. Wang J, Liu JH, Kurosawa T, Nada K, Ban Y, Moriguchi T: Cloning, biochemical identification, and expression analysis of a gene encoding $S$-adenosylmethionine decarboxylase in navel orange (Citrus sinensis Osbeck). J Hortic Sci Biotechnol 2010, 85:219-226.

47. Liu X, Wang Z, Wang L, Wu R, Phillips J, Deng X: LEA 4 group genes from the resurrection plant Boea hygrometrica confer dehydration tolerance in transgenic tobacco. Plant Sci 2009, 176:90-98.

48. Murashige T, Skoog F: A revised medium for rapid growth and bioassays with tobacco tissue culture. Physiol Plant 1962, 15:473-497.

49. Horsh RB, Fry JE, Hoffmann NL, Eichholtz D, Rogers SC, Fraley RT: A simple and general method for transferring genes into plants. Science 1985, 227:1229-1231.

50. Liu HY, Feng DR, Liu B, He YM, Wang HB, Wang JF: Studies on subcellular localization of MpASR in onion epidermal cells mediated by Agrobacterium (in Chinese). J Trop Subtrop Bot 2009, 17:218-222.

51. Liu JH, Nada K, Honda C, Kitashiba H, Wen XP, Pang XM, Moriguchi T: Polyamine biosynthesis of apple callus under salt stress: importance of the arginine decarboxylase pathway in stress response. J Exp Bot 2006, 57:2589-2599.

52. Liu JH, Inoue $H$, Moriguchi $\mathrm{T}$ : Salt stress-mediated changes in free polyamine titers and expression of genes responsible for polyamine biosynthesis of apple in vitro shoots. Environ Exp Bot 2008, 62:28-35.

53. Liang YC, Chen Q, Liu Q, Zhang W, Ding R: Exogenous silicon (Si) increases antioxidant enzyme activity and reduces lipid peroxidation in roots of salt-stressed barley (Hordeum vulgare L.). J Plant Physiol 2003, 160:1157-1164.

\section{doi:10.1186/1471-2229-10-230}

Cite this article as: Huang et al:: Overexpression of PtrABF gene, a bZIP transcription factor isolated from Poncirus trifoliata, enhances dehydration and drought tolerance in tobacco via scavenging ROS and modulating expression of stress-responsive genes. BMC Plant Biology $201010: 230$.

\section{Submit your next manuscript to BioMed Central and take full advantage of:}

- Convenient online submission

- Thorough peer review

- No space constraints or color figure charges

- Immediate publication on acceptance

- Inclusion in PubMed, CAS, Scopus and Google Scholar

- Research which is freely available for redistribution 